Journal of Fluid Mechanics

http://journals.cambridge.org/FLM

Journal of

Additional services for Journal of Fluid Mechanics:

Email alerts: $\underline{\text { Click here }}$

Subscriptions: $\underline{\text { Click here }}$

Commercial reprints: $\underline{\text { Click here }}$

Terms of use : $\underline{\text { Click here }}$

\title{
Aerated granular flow over a horizontal rigid surface
}

\section{EAMES and M. A. GILBERTSON}

Journal of Fluid Mechanics / Volume 424 / December 2000, pp 169 - 195

DOI: $10.1017 /$ S0022112000001920, Published online: 16 November 2000

Link to this article: http://journals.cambridge.org/abstract_S0022112000001920

How to cite this article:

I. EAMES and M. A. GILBERTSON (2000). Aerated granular flow over a horizontal rigid surface. Journal of Fluid Mechanics, 424, pp 169-195 doi:10.1017/S0022112000001920

Request Permissions : $\underline{\text { Click here }}$ 


\title{
Aerated granular flow over a horizontal rigid surface
}

\author{
By I. EAMES AND M. A. GILBERTSON ${ }^{2}$ \\ ${ }^{1}$ Department of Mechanical Engineering, University College London, Torrington Place, \\ London, WC1E 7JE, UK \\ ${ }^{2}$ Department of Mechanical Engineering, University of Bristol, \\ University Walk Bristol BS8 1TR, UK
}

(Received 5 July 1999 and in revised form 17 March 2000)

The effect of a vertical gas flow on the dynamics of a coulombic granular material moving over a horizontal rigid porous surface has been studied experimentally and theoretically. The presence of a fluidizing gas significantly alters the granular flow dynamics. When the gas velocity, $u_{g}$, is below the minimum fluidization velocity, $u_{m f}$, the effect of the gas is to reduce the angle of repose $\theta$ from the value measured in the absence of a gas flow. When material is poured from a point source onto a horizontal surface it forms a pile, which adjusts through episodic avalanching to a self-similar conical shape. Under these conditions, the development of the pile is determined by the local force balance on individual particles and its extent may be expressed in terms of the volume of particles added and the angle of repose. A volume of material is poured continuously from a point source onto a surface according to $Q t^{\alpha}$. Below the minimum fluidization velocity, a quasi-static description gives the encroachment distance of the granular pile as $l=\left(2 Q /(2 \pi / 3)^{n-1} \tan \theta\right)^{\frac{1}{n+1}} t^{\frac{\alpha}{n+1}}$ where $n=1$ for a planar release and $n=2$ for an axisymmetric release.

A continuum description of fluidized granular flow has been developed by vertically averaging the mass and momentum conservation equations and including the momentum exchange between the gas and granular flow. The bulk movement is driven along the ground by horizontal gradients of particle pressure and is resisted by a viscous drag force due to the particles moving horizontally through a vertical gas flow. Above the minimum fluidization velocity the character of the granular flow is significantly altered and takes on fluid-like properties. The model predicts the shape of the fluidized granular pile and that the encroachment distance grows as $l=\lambda_{n \alpha}\left(Q\left(u_{g}+u_{m f}\right) / \bar{\epsilon}\right)^{\frac{1}{n+2}} t^{\frac{\alpha+1}{n+2}}$, where $\bar{\epsilon}$ is the void fraction in the bed and $\lambda_{n \alpha}$ is a constant. Below the conditions for fluidization $\left(u_{g}<u_{m f}\right)$, the pile of granular material grows quasi-statically when $t>t_{*}$, where $t_{*} \sim\left(\bar{\epsilon}^{n+1} Q\left(u_{g}+u_{m f}\right) / \mu^{2+n}\left(u_{m f}-u_{g}\right)^{2+n}\right)^{\frac{1}{1+n-\alpha}}$ corresponds to the critical time when frictional forces are comparable to gradients of particle pressure and the drag force. Numerical solutions describing the granular flow are presented.

Experimental observations of the shape and extent of planar and axisymmetric granular flows when $\alpha=1$ compare well with theoretical predictions for various values of particle volume flux $Q$, time $t$, and gas flow rate $u_{g}$. The mathematical 
description of fluidized granular flows along rigid surfaces indicates a strong analogy with buoyancy-driven flows in a porous medium. This analogy permits extension of our description to include flows down slopes and the effect of internal stratification.

\section{Introduction}

Granular materials such as ores, agricultural grain, snow, and sand occur widely in nature and industry. The transport of particles along solid surfaces is often important. This can take place through the influence of gravity on the particles; however a significant number of these flows are mediated by a gas flowing through them. An example of where this takes place in industrial equipment is the operation of fast-fluidized beds where a powder is transported pneumatically in a closed loop under the influence of a gas flow passing through it. Particles may be deposited in horizontal sections of pipes and it is common practice to introduce by-pass gas along these sections so that the powder is fluidized and choking can be prevented. Geophysical granular flows are often driven by buoyancy forces down slopes and can represent a significant environmental hazard because the material, such as snow or ash, is generally dense and travelling at speed (Hopfinger 1983). Some features of the structure and behaviour of these flows suggest that they are fluidized through the entrainment of air, the vaporization of water, or the production of gas from hot rocks, which assists their propagation (Druitt 1998).

Flows of granular material can be complex owing to, for example, their scale, large particle size distributions, or irregular particle shape, and these factors may have a very important effect on a particular flow. In all cases the effect of a interstitial gas flow has a significant effect on the propagation of the granular material. The aim of this paper is to understand this effect when particles are released onto a rigid, horizontal surface through which there is a vertical gas flow (see figure 1). The complications described above that can occur in industrial and environmental flows are eliminated by working on a small scale and using a well-characterized powder. In tackling this problem, we are bringing together two bodies of work: granular flow dynamics and fluidization. The flow of granular materials down a slope when there is no external gas flow has been studied, e.g. Savage \& Hutter (1989), Johnson \& Jackson (1987), and is of practical interest for describing the motion of avalanches and pyroclastic flows. There is also a large body of work on fluidized beds, but only a small fraction has considered the motion of fluidized material moving down a slope, e.g. Nott \& Jackson (1992), Brinkert \& Davidson (1995), and these examples are limited to steady channel flows.

Investigation of the flow along a horizontal surface allows the driving effect of gravity to be isolated. A vertically averaged formulation for the granular flow is proposed, derived from the continuity and momentum equations and a consistent physical description of the fluidization process. A brief review of these physical processes within flowing powders is given in $\S 2$. In $\S 3$ we develop a mathematical description of cohesionless granular flows which includes momentum transfer from the gas to the particulate flow. Expressions for the size and shape of a pile of material below and above $u_{m f}$ are derived. The apparatus used and the experiments performed are described in $\S 4$. The mathematical description is tested against experimental results in $\S 5$ and general conclusions are drawn in $\S 6$. 


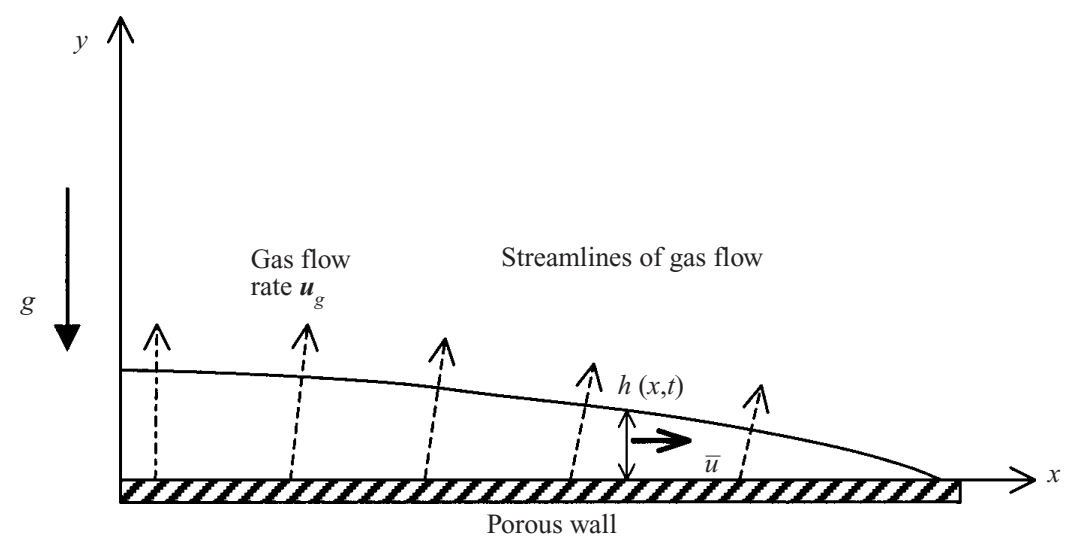

FIGURE 1. Schematic diagram showing a granular flow over a rigid horizontal surface, through which a gas flows.

\section{Physical processes within granular flows}

Four principal processes controlling a granular flow may be identified: cohesion, collisional and frictional interaction between particles, and interaction between the particles and the surrounding fluid. Cohesion may be important when inter-particle forces generated by static electricity, the presence of moisture or van der Waal's force, for example, are large compared with particle weight. The suppression of bubbling and uniform bed expansion above the point of minimum fluidization for fine material is intimately related to the strong inter-particle forces, which are significant for glass particles with diameters less than about $100 \mu \mathrm{m}$ (Molerus 1982). The particles used in the experiments below are significantly larger than this, and for the experimental arrangement described in $\S 4$, cohesive forces are negligible.

The collisional and frictional interaction between particles may be modelled at a microscopic level by a system of springs, dashpots, and sliders (e.g. Tsuji, Tanaka \& Ishida 1992), but the number of particles whose interaction may be described in this manner is limited, and the problem of incorporating the effect of interstitial fluid is still unclear. A wide range of continuum descriptions of granular flows has been proposed and for dense systems involving many particles the effects of interactions can be modelled as bulk properties of the powder. Two effects in particular may be considered to be important: inter-particle collisions due to the pseudo-thermal motion of the particles, and friction between particles.

\subsection{Inter-particle collisions}

Collisions between particles provide a means of transferring momentum and exerting an effective granular stress in the material. Many constitutive equations draw on an analogy with kinetic gas theory and only strictly apply when particle volume fractions are low. Lengthy expressions for the stress tensor analogous to those describing fluid motion result from this, and an extra equation is necessary for describing particle temperature, a measure of the strength of vibration of the particles. An authoritative derivation may be found in Lun et al. (1984).

\subsection{Inter-particle friction}

In addition to momentum transfer by inter-particle collisions, frictional forces provide another means of communicating momentum between adjacent particles. For dense 
flow friction can be modelled as a shear stress, that is dependent on normal stress. When the material is static the simplest description of shear is the coulomb yield criterion, which states that the shear stress $\tau$ varies linearly with $\sigma$, the normal stress, so that

$$
\tau=\mu \sigma+c,
$$

where $\mu$ is the coefficient of internal friction and $c$ is a yield stress describing cohesion. For coarse, cohesionless materials $c$ is negligible. The internal angle of friction $\phi$ is defined by

$$
\phi=\arctan \mu,
$$

and it can be shown that for a static, cohesionless, coulombic powder, $\phi$ is equal to the angle of repose. There are many empirical constitutive theories for frictional stress, but a powder undergoing plane shearing and fully developed is in a critical state, and all descriptions reduce to the Coulomb description (Johnson, Nott \& Jackson 1990).

\subsection{Fluidization}

When a vertical stream of gas is passed through a granular bed, the jump in gas pressure across the bed very often increases linearly with the gas velocity, $\boldsymbol{u}_{g}$ (see figure 2), because the drag exerted on the particles is dominated by viscous stresses, which are proportional to the gas flow. We shall call a bed in this state 'aerated'. When the gas flow is increased further, the point of minimum fluidization is reached where the entire weight of particles in the bed is just supported by the gas flow. At this point particle mobility is greatly increased, the bed may be easily sheared and superficially behaves as a fluid. One manifestation of this is that Archimede's Law appears to hold: a force is exerted on an immersed body equal to the mass of particles displaced. For example, a beaker partially filled with particles and placed in a fluidized bed will sink up to a level close to that of the particles in the beaker. Above the point of minimum fluidization, the pressure drop over the bed does not increase further and provided the particles are larger than a critical diameter (about $80 \mu \mathrm{m}$ for glass beads fluidized by air), bubbles are generated on the distributor plate and coalesce as they rise.

The superficial gas velocity corresponding to the point of minimum fluidization, $u_{m f}$, may be estimated by considering the balance between the weight of the bed and vertical drag. The average interstitial gas velocity through a packed bed of particles is $\boldsymbol{u}_{g} / \epsilon$, where $\epsilon$ is the voidage, so that the relative speed between the gas and the particles is $\left(\boldsymbol{u}_{g} / \epsilon-\boldsymbol{v}\right)$ where $\boldsymbol{v}$ is the particle velocity. The hydraulic radius is estimated from the ratio of the cross-sectional area of the flow to the wetted perimeter, $d_{p} \epsilon /(1-\epsilon)$, where $d_{p}$ is the surface-volume diameter of the particles. The Reynolds number based on interstitial flow is $R e=u_{g} d_{p} \rho_{g} / \epsilon \mu_{g}$, where $\mu_{g}$ is the fluid viscosity, and is typically less than 10 for the experiments described here, so drag is dominated by viscous stresses on the particle surface and the appropriate scaling for the drag per unit volume is $O\left(\mu_{g}\left|\boldsymbol{u}_{g} / \epsilon-\boldsymbol{v}\right|(1-\epsilon)^{2} / d_{p}^{2} \epsilon^{2}\right)$. Momentum exchange between the gas and particles under these conditions may be described by the first term of the semi-empirical Ergun equation to give

$$
\boldsymbol{f}_{g s}=\frac{150 \mu_{g}\left(\boldsymbol{u}_{g} / \epsilon-\boldsymbol{v}\right)(1-\epsilon)^{2}}{d_{p}^{2} \epsilon^{2}} .
$$

This expression differs from that proposed by Nott \& Jackson (1992), who expressed the drag in terms of the superficial difference of velocities rather than using the superficial gas velocity explicitly, but is identical to that proposed by Geldart (1986, 


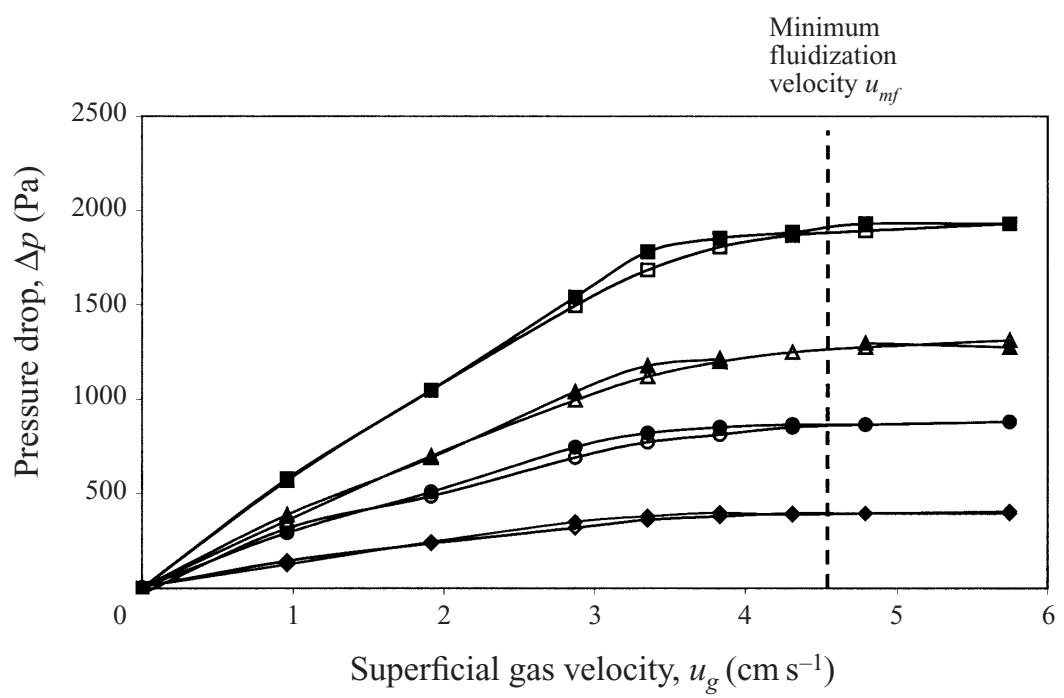

FIGURE 2. Pressure drop across a uniform bed of particles in a planar geometry as a function of gas flow rate for different bed depths: $13.2 \mathrm{~cm}(\boldsymbol{\nabla}), 9.4 \mathrm{~cm}(\boldsymbol{\Delta}), 6.6 \mathrm{~cm}(\bullet), 3.0 \mathrm{~cm}(\bullet)$. The closed symbols indicate pressure drop measurements as the gas velocity is increased and the open symbols denote measurements made as the gas velocity is decreased.

p. 21). When the material is fluidized, the drop in gas pressure across a bed of depth $h$ is equal to the weight of powder per unit area within it:

$$
\frac{\Delta p}{h}=(1-\epsilon)\left(\rho_{p}-\rho_{f}\right) g .
$$

When there is no mean flow of particles $(\boldsymbol{v}=\mathbf{0}), u_{m f}$ may be determined by balancing the pressure gradient (4) with the first term of the Ergun equation (3):

$$
u_{m f}=\frac{d_{p}^{2} \epsilon_{m f}^{3}\left(\rho_{p}-\rho_{g}\right) g}{150 \mu_{g}\left(1-\epsilon_{m f}\right)},
$$

where $\epsilon_{m f}$ corresponds to the voidage at the point of minimum fluidization (Davidson \& Harrison 1963).

The interactions between particles in a fluidized bed are usually expressed in terms of bulk quantities. In particular, a gas-fluidized bed is often treated as a bubbling Newtonian fluid and the effect of particle interaction is characterized by a viscosity. Measurements of viscosity have been made using a variety of methods involving several different types of viscometer or by inferring an apparent viscosity from the shape of rising bubbles (Grace 1970). However, there are significant difficulties associated with this (Brinkert \& Davidson 1995) and it is based on an over-simplified view of the nature of fluidized beds. Later, we shall demonstrate a closer analogy between fluidized flows over rigid surfaces and buoyancy-driven flows in porous media.

\subsection{The combination of physical mechanisms}

The relative importance of the different physical processes within granular flows, especially when fluidized, is a challenging area of research. One approach has been to use ad hoc expressions for pressure and viscosity in the stress tensor for the granular material. At its most crude fixed values are applied, but a more sophisticated approach, 
which met some success, was used by Anderson, Sundaresan \& Jackson (1995) for modelling bubble formation within fluidized beds. The form of the selected expressions and the parameters used accorded with the expected behaviour of the particles. An alternative approach, adopted by Johnson \& Jackson (1987) to model the plane shear flow of particles, is to build up the constitutive relations from the physical processes described previously. The stresses caused by pseudo-thermal motion and friction both result from direct interaction between particles, but they may be distinguished by duration of the contact: the pseudo-thermal motion results in brief collisions, while the frictional contribution is caused by longer sliding and rolling contacts (Nott \& Jackson 1992). It is unlikely that the two stresses are independent; however, to make the problem tractable Johnson \& Jackson (1987) calculated the total granular stress by simply adding the collisional stress $\boldsymbol{\sigma}_{s}$ and the frictional stress $\boldsymbol{\sigma}_{f}$ to give the so-called frictional-collisional model. This is a convenient, crude formulation which has the advantage of providing an accurate description in flows where either frictional or collisional stresses are dominant. Johnson et al. (1990) applied their description to particulate flows down inclined slopes and comparison with experiment showed that many of the qualitative characteristics of the flow were reproduced by the model. Nott \& Jackson (1992) examined granular flows down slopes through which gas flowed up to speeds just beyond the point of minimum fluidization. This required the addition of a term in the momentum equation to describe momentum exchange between the particles and aerating gas, although crucially the component of drag parallel to the wall (which plays an essential role in our description) is absent from their final formulation. Their model was sensitive to a number of empirical parameters, such as a specularity constant.

Both $\boldsymbol{\sigma}_{s}$ and $\boldsymbol{\sigma}_{f}$ can be significant in granular flows. By comparing different descriptions of the behaviour of a powder moving down an inclined plane, Anderson \& Jackson (1992) found that it could only be described realistically when friction is included. The relative importance of collisional stresses compared with friction in an aerated bed depends upon the degree of support of the particles, the particle volume fraction, and the shear rate. In the experiments described in this paper, the void fraction is close to packing density so that collisional stresses are negligible, and the total granular stress is dominated by frictional stress $\boldsymbol{\sigma}_{f}$. This may be further justified through a scaling analysis of the pseudo-thermal energy equation. It can be shown that production of pseudo-thermal stress by the wall is small compared to the production by the fluidizing gas flow under similar conditions to those in the experimental study, and the effect of thermal stress production is negligible compared to drag and frictional components. This argument is presented in Appendix A.

\section{Mathematical model of aerated and fluidized granular flows}

The effect of a fluidizing gas on a granular flow along a horizontal surface can be described by a continuum model developed by vertically averaging the mass and momentum equations describing particulate flow. This approach was applied by Savage \& Hutter (1989) to describe coulombic material flowing down rough walls, but the additional effect of the momentum exchange between the gas and particles is included in this new description, which is tested experimentally.

The mass conservation equation describing granular flow is

$$
-\frac{\partial \epsilon}{\partial t}+\nabla \cdot((1-\epsilon) \boldsymbol{v})=0,
$$


where $\epsilon$ is the void fraction. The momentum conservation equation describing the particle phase is

$$
\rho_{p}(1-\epsilon) \frac{\mathrm{D} \boldsymbol{v}}{\mathrm{D} t}=\left(\rho_{p}-\rho_{g}\right)(1-\epsilon) \boldsymbol{g}-\nabla p_{s}+\boldsymbol{f}_{g s}+\nabla \cdot \boldsymbol{\sigma},
$$

where $\rho_{p}$ is the density of the granular material, $\boldsymbol{g}$ is gravitational acceleration, $\boldsymbol{f}_{g s}$ is the momentum exchange between the gas and particles, and we define $\sigma$ as the difference between the total stress and $-p_{s} I$. The particle pressure $p_{s}$ is analogous to the hydrostatic pressure in a liquid and is proportional to the weight of the particles,

$$
p_{s}(y)=(h-y)\left(\rho_{p}-\rho_{g}\right)(1-\epsilon) g .
$$

This term is important because it is the gradient of particle pressure along the rigid surface that induces the material to flow. The rate of exchange of momentum between the particles and the fluidizing gas is $\boldsymbol{f}_{g s}$ and is described by (3). The gas travels along the path of least resistance from the rigid surface (the distributor plate) to the free surface. The granular flow along the horizontal rigid surface is dense and negligible dilation was observed when bubbles were not present. As discussed above, the collisional stress is negligible compared to the total stress, which is dominated by a frictional component. The granular material is coulombic, so the tangential frictional stress at the wall is proportional to the normal stress, $N_{f}$, which is equal to the effective weight per unit area of the particles supported on the rigid surface:

$$
N_{f}=\left[\left(\rho_{p}-\rho_{g}\right)(1-\bar{\epsilon}) g-\boldsymbol{f}_{g s} \cdot \hat{\boldsymbol{y}}\right] h,
$$

where $\hat{\boldsymbol{y}}$ is a unit vector normal to the rigid surface. For sloping layers of granular material, the layer of material is aerated when

$$
\left(\rho_{p}-\rho_{g}\right)(1-\bar{\epsilon}) g-\boldsymbol{f}_{g s} \cdot \hat{\boldsymbol{y}}>0,
$$

and this condition reduces to $u_{g}<u_{m f}$ for a uniform layer of particles.

Equation (9) shows that as the gas flow rate increases, a larger fraction of the weight of the particles is supported by the gas, and this results in a larger drop in gas pressure across the bed. When the material is fully fluidized, the bed of particles is supported by gas, and according to (9) the normal stress on the rigid surface is zero. In practice the reaction on the wall is not identically zero because the pressure drop across the bed fluctuates owing to the generation of bubbles on the distributor plate and their eruption at the free surface. However this component is not included in our description and we prescribe

$$
N_{f}=0,
$$

when the material is fluidized and $\left(\rho_{p}-\rho_{g}\right)(1-\bar{\epsilon}) g-\boldsymbol{f}_{g s} \cdot \hat{\boldsymbol{y}}<0$. Equations (6) and (7) are solved subject to the stress conditions

$$
\hat{\boldsymbol{y}} \cdot \boldsymbol{\sigma} \cdot \hat{\boldsymbol{x}}+\mu N_{f}=0
$$

on the rigid surface and

$$
\hat{\boldsymbol{y}} \cdot \boldsymbol{\sigma}=\mathbf{0}
$$

perpendicular to the free surface.

\subsection{Vertically averaged long-wave formulation}

A vertically averaged formulation may now be developed based on the assumption that the variation in the granular depth $h$ is small over horizontal distances comparable to the bed depth $(|\partial h / \partial x| \ll 1)$. In a fluidized system we anticipate significant slip 
between the bulk material and the rigid surface, so that the flow is approximately uniform with depth. Vertically averaging the equation describing the conservation of mass yields

$$
\frac{\partial h}{\partial t}+\frac{1}{x^{n-1}} \frac{\partial}{\partial x}\left(x^{n-1} h \bar{u}\right)=0,
$$

where $n=1,2$ for planar and axisymmetric flows respectively, and $x$ represents the planar or radial distance from the source of material, and $\bar{u}$ is depth-averaged velocity. Integrating the horizontal momentum equation vertically and applying the stress boundary conditions (12) and (13) yields

$$
\rho_{p}(1-\bar{\epsilon})\left(\frac{\partial \bar{u}}{\partial t}+\bar{u} \frac{\partial \bar{u}}{\partial x}\right)=-\left(\rho_{p}-\rho_{g}\right)(1-\bar{\epsilon}) g \frac{\partial h}{\partial x}-\frac{1}{h} \mu N_{f}+\boldsymbol{f}_{g s} \cdot \hat{\boldsymbol{x}},
$$

See Appendix B for details of the derivation of equations (14) and (15). Averaging the vertical component of the momentum equation across the depth of the granular layer yields equation (9).

The gas travels along the path of least resistance from the distributor plate to the free surface. The gas flow adjusts rapidly to the evolving granular flow providing the time taken for gas to travel from the porous plate to the free surface, $h / u_{g}$, is much shorter than the advective time scale $L / \bar{u}$ associated with the granular flow (where $L$ is the horizontal length scale associated with the pile). Moreover, under the long-wave restriction $(|\partial h / \partial x| \ll 1)$ the gas flow will travel the shortest distance from the wall to the granular surface so that

$$
\boldsymbol{u}_{g} \times \hat{\boldsymbol{n}}=\mathbf{0},
$$

where $\hat{\boldsymbol{n}}=-\hat{\boldsymbol{x}} \sin \beta+\hat{\boldsymbol{y}} \cos \beta$ is normal to the surface of the granular layer. Under the restriction $h / L \ll u_{g} / \bar{u}$ the drag on the granular flow is therefore

$$
\mathbf{f}_{g s}=\frac{150 \mu_{g}(1-\bar{\epsilon})^{2}}{d_{p}^{2} \bar{\epsilon}^{2}}\left(\left(-\bar{u}-\frac{u_{g}}{\bar{\epsilon}} \sin \beta\right) \hat{\boldsymbol{x}}+\frac{u_{g}}{\bar{\epsilon}} \cos \beta \hat{\boldsymbol{y}}\right) .
$$

The dominant terms in (15) are identified by estimating the relative magnitude of the inertia, pressure gradient, friction and drag forces. Denoting the characteristic velocity and vertical length scales by $U$ and $H$ respectively, the estimated magnitudes of individual terms are

$$
\begin{aligned}
& \underbrace{\rho_{p}(1-\bar{\epsilon})\left(\frac{\partial \bar{u}}{\partial t}+\bar{u} \frac{\partial \bar{u}}{\partial x}\right)}_{O\left(\frac{\rho_{p} U^{2}}{L}\right)}=\underbrace{-\left(\rho_{p}-\rho_{g}\right)(1-\bar{\epsilon}) g \frac{\partial h}{\partial x}}_{O\left(\frac{\rho_{p} g H}{L}\right)} \\
& \begin{array}{cc}
-\underbrace{\frac{1}{h}}_{\left(\frac{\rho_{p} g\left|u_{m f}-u_{g}\right|}{u_{m f}}\right)} & O\left(\frac{\rho_{p} g\left|U-u_{g}\right|}{u_{m f}}\right)
\end{array}
\end{aligned}
$$

The relative magnitude of inertia and the frictional and drag forces is characterized by the dimensionless parameter $\mathscr{R}$, where

$$
\frac{1}{\mathscr{R}}=\frac{L g}{U^{2}} \max \left(\frac{\left|u_{m f}-u_{g}\right|}{u_{m f}}, \frac{\left|U-u_{g}\right|}{u_{m f}}\right) .
$$

The parameter $\mathscr{R}$ plays an analogous role to the Reynolds number in fluid flows. 
When $\mathscr{R} \ll 1$ the flow is described by a balance between the frictional and drag forces, which retard the granular flow, and horizontal gradients of particle pressure, which drives the flow along the wall. In the opposing limit $\mathscr{R} \gg 1$, the balance is between the inertial force and gradients in particle pressure and this limit corresponds closely to the problem described by Savage \& Hutter (1989) for the release of dense material down a slope. In our experimental study, we estimate $\mathscr{R}$ to be less than $10^{-2}$ and so describe the limiting case $\mathscr{R} \ll 1$ applicable to our experimental observations.

In the limit $\mathscr{R} \ll 1$, the granular flow is determined by a balance between horizontal gradients of particle pressure and friction, and the horizontal component of drag arising from the gas flow:

$$
0=-\left(\rho_{p}-\rho_{g}\right)(1-\bar{\epsilon}) g \frac{\partial h}{\partial x}-\mu\left[\left(\rho_{p}-\rho_{g}\right)(1-\bar{\epsilon}) g-\boldsymbol{f}_{g s} \cdot \hat{\boldsymbol{y}}\right]+\boldsymbol{f}_{g s} \cdot \hat{\boldsymbol{x}} .
$$

When the material is aerated and (10) is satisfied, equation (20) may be rearranged to give

$$
\begin{aligned}
& \underbrace{\bar{u}}_{O\left(\frac{L}{t}\right)}=\underbrace{-\frac{1}{\bar{\epsilon}}\left(\frac{u_{g}}{\left(1-\left(\frac{\partial h}{\partial x}\right)^{2}\right)^{1 / 2}}+u_{m f} \frac{\left(1-\epsilon_{m f}\right) \bar{\epsilon}^{3}}{(1-\bar{\epsilon}) \epsilon_{m f}^{3}}\right) \frac{\partial h}{\partial x}}_{O\left(\frac{u_{g}+u_{m f}}{\bar{\epsilon}} \frac{H}{L}\right)} \\
& +\underbrace{\frac{\mu}{\bar{\epsilon}}\left(\frac{u_{g}}{\left(1-\left(\frac{\partial h}{\partial x}\right)^{2}\right)^{1 / 2}}-u_{m f} \frac{\left(1-\epsilon_{m f}\right) \bar{\epsilon}^{3}}{(1-\bar{\epsilon}) \epsilon_{m f}^{3}}\right)}_{O\left(\frac{\mu\left(u_{g}-u_{m f}\right)}{\bar{\epsilon}}\right)} .
\end{aligned}
$$

When the material is fluidized and (10) is not satisfied, the second term on the righthand side of (21), which corresponds to the frictional stress term, is zero because the particles are supported by a vertical stream of gas.

Scaling analysis may now be applied to determine the appropriate dynamic balance of terms in (21). The volume of granular material added to the system is $Q t^{\alpha} \sim L^{n} H$, where $\alpha$ is a constant describing the mode of release of the material. When frictional stresses are negligible, the first term on the right-hand side of (21) describing fluid drag may be balanced with the horizontal pressure gradients driving the flow along the wall so that the encroachment length grows as

$$
L \sim Q^{\frac{1}{n+2}} t^{\frac{\alpha+1}{n+2}}\left(\left(u_{g}+u_{m f}\right) / \bar{\epsilon}\right)^{\frac{1}{n+2}} .
$$

The relative strength of the frictional forces, compared to the pressure gradient, increases with time as $H / L$ decreases (according to (21)) so that after a critical time $t_{*}$, frictional forces may no longer be neglected. The characteristic time when frictional 
forces and the pressure gradient terms are comparable occurs when

$$
\frac{\text { frictional forces }}{\text { pressure gradient and drag }} \sim \mu \frac{u_{g}-u_{m f}}{u_{g}+u_{m f}} Q^{\frac{-1}{n+2}} t_{*}^{\frac{n+1-x}{n+2}}\left(\frac{u_{g}+u_{m f}}{\bar{\epsilon}}\right)^{\frac{n+1}{n+2}} \sim 1,
$$

whence

$$
t_{*} \sim\left(\frac{Q \bar{\epsilon}^{n+1}}{\mu^{n+2}} \frac{u_{m f}+u_{g}}{\left(u_{m f}-u_{g}\right)^{n+2}}\right)^{\frac{1}{n+1-\alpha}} .
$$

When $t \ll t_{*}$, an aerated granular material flows according to the scaling analysis developed for the fully fluidized material given by (22). However, for $t \geqslant t_{*}$, frictional stresses are important and the flow quickly develops according to a quasi-static balance between friction and horizontal gradients of particle pressure. In the context of the experimental study, $t_{*}$ has a typical value of $O(2-5) \mathrm{s}$ when $u_{g}=0$, depending on the value of $Q$ and $n$. This is much less than the characteristic time taken for the granular layer to reach the end of the tank, indicating that the granular flows observed are dominated by frictional stresses and the quasi-static description is applicable. When $u_{g} / u_{m f}$ is increased above about 0.5 , the value of $t_{*}$ increases rapidly. Close to the minimum point of fluidization the time taken for the granular flow to propagate the length of the tank, is less than $t_{*}$, and a quasi-static description is not applicable.

\subsection{Quasi-static model for $u_{g} / u_{m f}<1$ and $\mathscr{R} \ll 1$}

When $u_{g} / u_{m f}<1$ and $t \geqslant t_{*}$, the granular pile grows quasi-statically because the adjustment of the pile occurs more quickly than the rate at which material is introduced on the wall. The quasi-static growth is determined by substituting $\bar{u}=0$ into (21). Equation (21) indicates that the gradient of the pile is independent of the depth of the layer and so is constant. The angle to the horizontal, $\theta$, may be found from (21) using $\partial h / \partial x=-\tan \theta$ to obtain

$$
\tan \theta=\mu\left(\frac{u_{m f} \bar{\epsilon}^{3}\left(1-\epsilon_{m f}\right)-\left(u_{g} \epsilon_{m f}^{3}(1-\bar{\epsilon})\right) \cos \theta}{u_{m f} \bar{\epsilon}^{3}\left(1-\epsilon_{m f}\right)+\left(u_{g} \epsilon_{m f}^{3}(1-\bar{\epsilon})\right) \cos \theta}\right) .
$$

When $u_{g}=0$, the slope angle corresponds to the angle of repose $\phi$, and in the limit of incipent fluidization the slope angle tends to zero because the frictional stress is not capable of supporting horizontal gradients of particle pressure.

Implicit in our continuum description is that the pile adjusts through bulk movement when aerated. A static two-dimensional pile confined between rigid walls is unable to adjust through bulk movement owing to frictional forces on the walls, and adjustment occurs by particles sliding down the surface of the pile. This behaviour may be understood by considering the forces on individual particles lying on the surface of the granular pile. Such an approach was successfully used by Gilbertson \& Yates (1996) to describe the behaviour of tilted fluidized beds. The gas flow will tend to take the shortest path from the distributor plate to the surface of the granular pile, and will therefore be normal to the granular surface. It will exert a drag force on the particles, reducing the reaction of the particle to the surface and therefore reducing the frictional force that prevents it sliding. The component of the reaction force, $R$, perpendicular to the surface of the pile is the difference between the component of particle weight in that direction and the drag force:

$$
R=\frac{1}{6} \pi\left(\rho_{p}-\rho_{f}\right) g d_{p}^{3} \cos \theta-\frac{1}{8} \rho_{f} C_{D}(R e) \pi d_{p}^{2} u_{g}\left|u_{g}\right|,
$$


where $C_{D}$, the drag coefficient, is a function of gas flow rate, particle volume fraction and particle diameter.

Particle slippage occurs when the component of weight down the slope is balanced by friction, or

$$
m g \sin \theta=\mu R \text {. }
$$

Combining equations (25) and (26) yields

$$
\phi-\theta=\sin ^{-1}\left(\frac{C_{D}(R e) u_{g}\left|u_{g}\right|}{C_{D}\left(R e_{m f}\right) u_{m f}^{2}} \sin \phi\right)
$$

where $R e_{m f}$ is the particle Reynolds number at the point of minimum fluidization. The two limiting cases of $\theta \rightarrow 0$ as $u_{g} \rightarrow u_{m f}$ and $\theta \rightarrow \phi$ as $u_{g} \rightarrow 0$ are satisfied by (27). Physically (27) describes when the mode of adjustment is by particle slippage down the surface of the granular pile and (24) describes adjustment by bulk movement of the pile. An interesting result of the above equations is that sucking gas through the granular pile stabilizes the slope so that $\theta \geqslant \phi$.

When material is poured onto a horizontal surface, we observed that the granular pile adjusts by episodic avalanching in a planar geometry or through continuous avalanching, sometimes precessing around the apex of a conic pile, for axisymmetric releases. The adjustment was quasi-static in the sense that the material stopped flowing when pouring ceased. When the volume of material released is $Q t^{\alpha}$, conservation of mass requires

$$
Q t^{\alpha} \frac{1-\epsilon_{0}}{1-\bar{\epsilon}}=\int_{0}^{l} h(x, t)(2 \pi x)^{n-1} \mathrm{~d} x=\frac{1}{2}\left(\frac{2 \pi}{3}\right)^{n-1} l^{n} h .
$$

The void fraction in the pile, $\bar{\epsilon}$, is a function of gas flow rate and may differ from the voidage $\epsilon_{0}$ prior to pouring. The horizontal or radial extent of the granular pile can then be shown to be

$$
l=\left(\frac{2 Q\left(\frac{1-\epsilon_{0}}{1-\bar{\epsilon}}\right)}{\left(\frac{2}{3} \pi\right)^{n-1} \tan \theta}\right)^{\frac{1}{n+1}} t^{\frac{\alpha}{n+1}},
$$

where the condition that the slope has a constant gradient is applied. In the experimental study described later, powder is poured at a constant rate $(\alpha=1)$, and as apparent dilation was negligible, it will be assumed that $\epsilon_{0}=\bar{\epsilon}$. It is important to note that close to the fluidization velocity $\left(u_{g} / u_{m f} \rightarrow 1\right)$, the critical time $t_{*}$ increases rapidly and time scales characterizing the adjustment of the granular pile and the rate at which material is introduced are comparable. Under this condition, the quasi-static description is no longer applicable, and a dynamic description of this motion must be developed.

\subsection{Dynamic model for $\mathscr{R} \ll 1$}

When the material is fluidized $\left(u_{g} / u_{m f}>1\right)$, the weight of the particles is supported by the gas flow and $N_{f}=0$ in accordance with our mathematical description. As the fluidized granular flow propagates, the gradient of the granular flow decreases with time and we proceed to develop analytical solutions describing the flow for $t \ll t_{*}$, under the long-wave approximation $(|\partial h / \partial x| \ll 1)$ when equation $(21)$, describing the 
propagation of the granular flow, reduces to

$$
\bar{u}=-\frac{1}{\bar{\epsilon}}\left(u_{g}+u_{m f} \frac{\left(1-\epsilon_{m f}\right) \bar{\epsilon}^{3}}{(1-\bar{\epsilon}) \epsilon_{m f}^{3}}\right) \frac{\partial h}{\partial x} .
$$

Equation (30) may be coupled with the equation for the conservation of mass (14) to obtain a nonlinear diffusion equation describing the dynamics of a fluidized granular flow:

$$
\frac{\partial h}{\partial t}=\frac{1}{x^{n-1}} \frac{\partial}{\partial x}\left(x^{n-1} \frac{1}{\bar{\epsilon}}\left(u_{g}+u_{m f} \frac{\left(1-\epsilon_{m f}\right) \bar{\epsilon}^{3}}{(1-\bar{\epsilon}) \epsilon_{m f}^{3}}\right) h \frac{\partial h}{\partial x}\right) .
$$

The above equation may be solved in conjunction with the condition that the volume of granular material poured onto the surface is

$$
\int_{0}^{l} h(x, t)(2 \pi x)^{n-1} \mathrm{~d} x=Q t^{\alpha} \frac{1-\epsilon_{0}}{1-\bar{\epsilon}}
$$

where $l$ denotes the lateral extent of the pile. Nonlinear diffusion equations such as (31) are found to describe a wide range of fluid phenomena and are solved using standard analytical and numerical methods. For instance, Huppert \& Woods (1995) studied two-dimensional buoyancy-driven flows in porous media and under the condition that the permeability of the porous media is uniform, their set of equations reduces to (31) (see Bear 1988). Similarity solutions to (31) may be constructed in terms of similarity variables so that $h(x, t)=H \tilde{h}(\tilde{x}, t)$ where $\tilde{x}=x / L$. The horizontal and vertical length scales, $H$ and $L$ respectively, are determined by balancing terms in (31) and terms in (32). This generates two simultaneous equations which may be solved to derive the appropriate scalings for $H$ and $L$. The similarity solution reduces the nonlinear partial differential equation (31) to a nonlinear ordinary differential equation

$$
\frac{(2+n)}{\tilde{x}^{n-1}} \frac{\mathrm{d}}{\mathrm{d} \tilde{x}}\left(\tilde{x}^{n-1} \tilde{h} \frac{\mathrm{d} \tilde{h}}{\mathrm{~d} \tilde{x}}\right)=(2 \alpha-1) \tilde{h}-(\alpha+1) \tilde{x} \frac{\mathrm{d} \tilde{h}}{\mathrm{~d} \tilde{x}},
$$

which may be solved analytically or numerically. The length of the fluidized pile is proportional to the horizontal length scale $L, l=\lambda_{n \alpha} L$, where the coefficient $\lambda_{n \alpha}$ depends on $\alpha$ and $n$ and is determined uniquely from the condition $\tilde{h}\left(\lambda_{n \alpha}\right)=0$ and from the constraint (32) which now reduces to $\int_{0}^{\lambda_{n x}}(2 \pi \tilde{x})^{n-1} \tilde{h}(\tilde{x}) \mathrm{d} \tilde{x}=1$. The lateral extent of the granular pile is

$$
l=\frac{\lambda_{n \alpha}}{\bar{\epsilon}^{\frac{1}{n+2}}}\left(u_{g}+u_{m f} \frac{\left(1-\epsilon_{m f}\right) \bar{\epsilon}^{3}}{(1-\bar{\epsilon}) \epsilon_{m f}^{3}}\right)^{\frac{1}{n+2}}\left(\frac{Q\left(1-\epsilon_{0}\right)}{(1-\bar{\epsilon})}\right)^{\frac{1}{n+2}} t^{\frac{\alpha+1}{n+2}} .
$$

The coefficient $\lambda_{n \alpha}$ may be determined numerically by solving (33); for a constant flux release of particles, $\alpha=1$, we obtain $\lambda_{11}=1.48$ and $\lambda_{21}=1.15$.

The shape of a fluidized planar pile $(n=1)$ is described by a series expansion:

$$
\frac{h}{H}=\frac{\lambda_{1 \alpha}^{2}(\alpha+1)}{3}\left(1-\frac{x}{l}\right)+\frac{\lambda_{1 \alpha}^{2}(\alpha-2)}{12}\left(1-\frac{x}{l}\right)^{2}-\frac{\alpha \lambda_{1 \alpha}^{2}}{72} \frac{(\alpha-2)}{(\alpha+1)}\left(1-\frac{x}{l}\right)^{3}+\cdots .
$$

When an instantaneous volume of material is released $(\alpha=0)$, the solution to (35) is given exactly by the first two terms of the above expansion. When $\alpha=1$ the difference between the numerical calculation of the shape profile and the expansion truncated after the third term is less than $1 \%$. The shape profile for an axisymmetric flow is obtained from the numerical solution of (33). 
When the material is aerated $\left(u_{g} / u_{m f}<1\right)$ and $t \ll t_{*}$, the granular flow in planar and axisymmetric geometries is calculated numerically by solving a nonlinear advection/diffusion equation describing the evolution of the height of the granular pile, which is obtained by substituting (21) into (14). The numerical solutions are obtained by using a finite difference scheme explicit in time, and using a small time step. The simulations were repeated using finer mesh sizes and time increments to establish convergence. At each location, the criterion (10) was applied to determine whether the layer was locally fluidized or aerated.

\section{Experimental arrangement}

We have developed a quasi-static description of granular flows corresponding to $u_{g} / u_{m f}<1$, and a dynamic description when the material is fluidized or $u_{g} / u_{m f}>1$. When $u_{g} / u_{m f}<1$, the lateral extent of the pile is described by (29), and the angle of repose by (24) when adjustment occurs through bulk movement or (27) when adjustment occurs through avalanching. When $u_{g} / u_{m f}>1$, the lateral extent of the pile is described by (34) and its shape from (35). In the absence of bubbles, the dilation of the powder was negligible and the assumption $\bar{\epsilon}=\epsilon_{0}=\epsilon_{m f}$ applies. To test these models a series of small-scale experiments was performed where the size and shape of flows of aerated granular material were observed and measured.

The experiments were carried out in Perspex containers whose floors consisted of porous sintered plastic sheeting $(10 \mathrm{~mm}$ thick Vyon D sheeting from Porvair Ltd) through which gas was passed. Two tank geometries were employed: a planar tank $291 \mathrm{~mm}$ wide with walls separated by $6 \mathrm{~mm}$, and a cylindrical tank of diameter $290 \mathrm{~mm}$. Particles were introduced into the containers through different sized funnels according to the particle mass flux desired. The mass flux of particles through a funnel is independent of the depth of powder within it (Nedderman 1992), and this was verified experimentally for our system. The particles used were glass spheres, $90 \%$ of which had diameters within the range of 212 and $250 \mu \mathrm{m}$, and of density $2500 \mathrm{~kg} \mathrm{~m}^{-3}$ (Potters Ballotini grade AD). These particles may be classified as a Type B powder in the Geldart classification, which corresponds to the property that dilation of the powder is negligible for $u_{g} / u_{m f}<1$ and the bed bubbles for $u_{g} / u_{m f}>1$. The motion of the particle flows was recorded on S-VHS videotape. Measurements were made either directly from the images or they were captured from the tape using a Data Translations DT3155 card and analysed using the UTHSCSA ImageTool program.

The variation of pressure drop with superficial gas speed for the planar beds is shown in figure 2 for different bed depths. The boundary between a packed bed and a wholly fluidized state is not sharply defined and there is some smoothing of the curve near the point of minimum fluidization. Conventionally $u_{m f}$ is taken as the intersection between the straight lines drawn through the aerated and the fluidized portions of the pressure drop-gas velocity curve. We use a more physical definition of $u_{m f}$ as the gas velocity at which the entire weight of the bed is supported, i.e. the velocity at which the curves in figure 2 become level. This corresponds to $u_{m f}=4.53 \mathrm{~cm} \mathrm{~s}^{-1}$ for the planar beds and this value is independent of bed depth. The value of $u_{m f}$ measured in the axisymmetric container is $5.75 \mathrm{~cm} \mathrm{~s}^{-1}$. Both measurements lie in the range $4.4-6.5 \mathrm{~cm} \mathrm{~s}^{-1}$ predicted by (5).

Two sets of experiments were performed. In the first series the effect of gas flow on the angle of repose of a granular pile was examined. These quasi-static experiments permitted the particles to be characterized and demonstrated the effect of gas flow 
on friction between particles in the bed. The angle of repose of a fixed volume of granular material was measured in both geometries. The particles were poured through a funnel onto a horizontal porous surface to form a conical pile. The air flow through the pile was increased incrementally and the shape recorded until the powder was fluidized. The angle of repose was measured directly from recorded images and found to be independent of the volume of powder introduced onto the wall. In the second series of experiments, the effects of gas flow on the dynamic behaviour of the particles were examined. Particles were introduced at a constant flux directly onto the porous base of a container through which gas was being passed. The development with time of the resulting flow of particles was then observed and recorded.

\section{Experimental results and comparison with theory}

\subsection{The angle of repose of fixed volumes of particles}

When a granular material is poured onto a horizontal surface, the processes which form the resulting pile of material depend upon the degree of friction between the particles. When friction is high the pile develops through a series of avalanches until equilibrium between the weight of the powder and friction between the particles is reached. This results in a cone growing as a series of laminar layers and may be described through the forces acting on a single particle giving rise to equation (27). When interparticle friction is low, bulk movement of particles takes place as described by equation (24). Both modes of adjustment generate a conical pile of material whose angle of repose is uniquely related to the amount of friction between particles. When the bed is aerated, internal friction is reduced owing to the partial support of particles by the gas flow, and so a smaller angle of repose is observed than in the absence of a gas flow. When the particles are fully fluidized, the friction between them is reduced to such an extent that the pile is unable to support internal stresses and the bed adjusts until its surface is level.

Figure 3(a) shows the measured angles of repose for a planar bed when it is aerated compared with theoretical predictions for when the bulk movement of particles takes place, equation (24), and when a force balance on a single particle close to the surface of the pile is used, equation (27). Two lines have been plotted for the behaviour described by equation (27), one corresponding to a low-Re description of drag force for which $C_{D}\left(R e_{p}\right) u_{g} / C_{D}\left(R e_{m f}\right) u_{m f}=1$ and one to a high-Re description of drag force for which $C_{D}\left(R e_{p}\right) / C_{D}\left(R e_{m f}\right)=1$. There is good agreement between the experimental measurements and the calculation of $\theta$ based on the high-Re version of equation (27). This is surprising as the Reynolds number is expected to be small $\left(R e_{m f} \sim 2\right)$; however the model does not account for a number of features such as the effect of surrounding particles. Nevertheless, agreement is sufficiently good to suggest that the behaviour of the particles in the planar case is controlled by the local balance of forces on the surface of the pile, and the avalanching that results from it.

Similar measurements of the angle of repose for an axisymmetric pile of granular material are compared in figure $3(b)$ with theoretical predictions based on the same cases as in figure 3(a). These observations are in better agreement with (24) than (27), suggesting that in the absence of the restraining influence of the walls, the particles largely move as a bulk material rather than as individuals close to the surface. This is what appeared to take place in the experiments. 

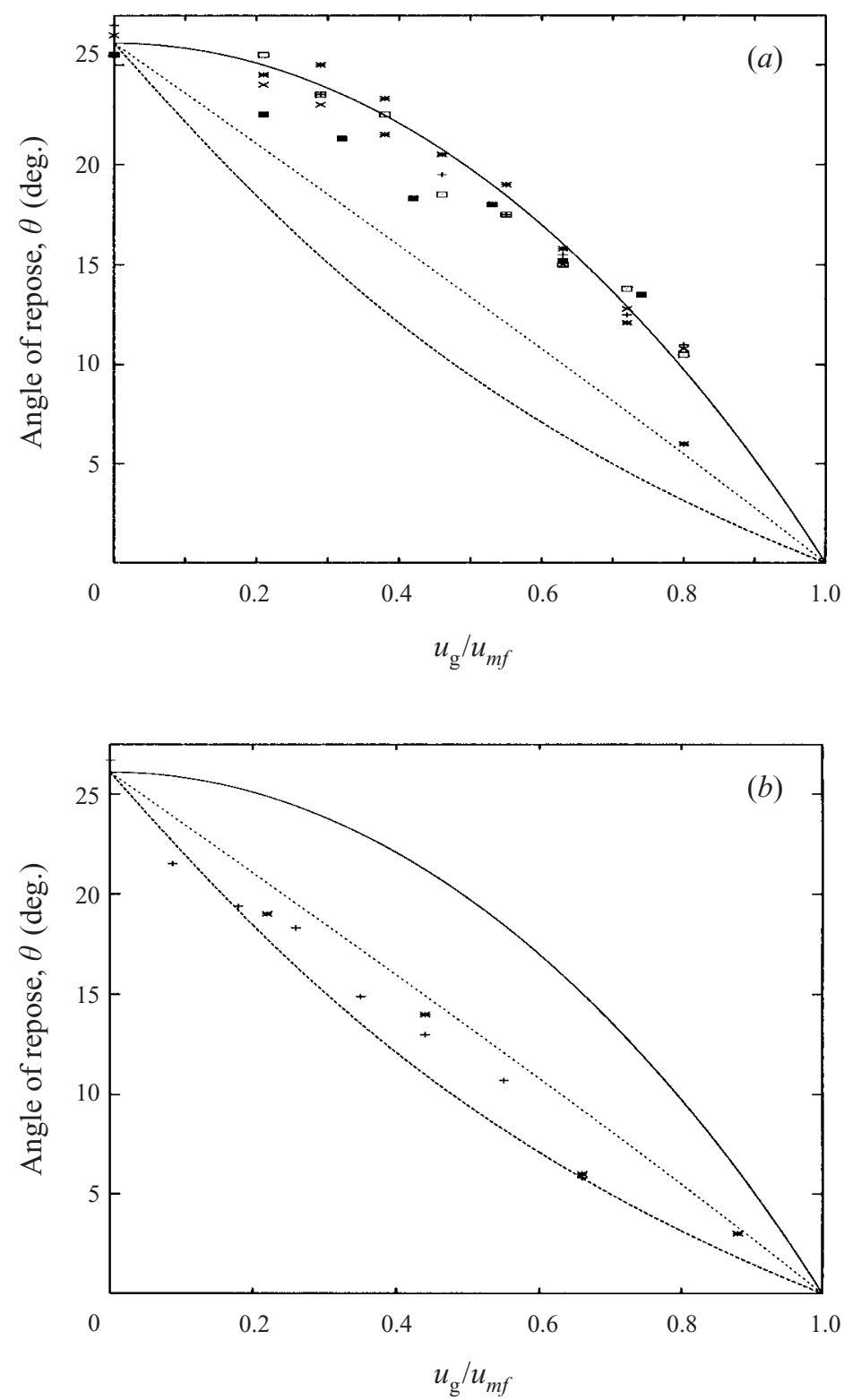

FIGURE 3. Measured angle of repose as a function of gas flow rate for aerated granular piles for $(a)$ Planar and $(b)$ axisymmetric release of material. The symbols denote volume of particles added: in $(a) \square, 26.3 \mathrm{ml} ; \times, 115.6 \mathrm{ml} ; \mathbf{\square}, 234.8 \mathrm{ml} ;+, 164.7 \mathrm{ml} ; *, 52.3 \mathrm{ml}$; and in $(b)+, 100 \mathrm{ml} ; \times$, $50 \mathrm{ml}$. The full and dotted curves correspond to prediction by $(27)$, where $C_{D}(\operatorname{Re}) / C_{D}\left(\operatorname{Re} e_{m f}\right)=1$, $C_{D}(R e) u_{f} / C_{D}\left(R e_{m f}\right) u_{m f}=1$ respectively; the dashed curve corresponds to (24). The angle of repose in the absence of gas flow is $\phi=26.1^{\circ}$.

\subsection{Shape and dynamics of granular flows}

We have observed that as the gas flow rate is increased the conical shape of the pile of granular material is distorted, partly because it can encourage different bulk behaviour in different regions of a pile of material. This change is most clearly 


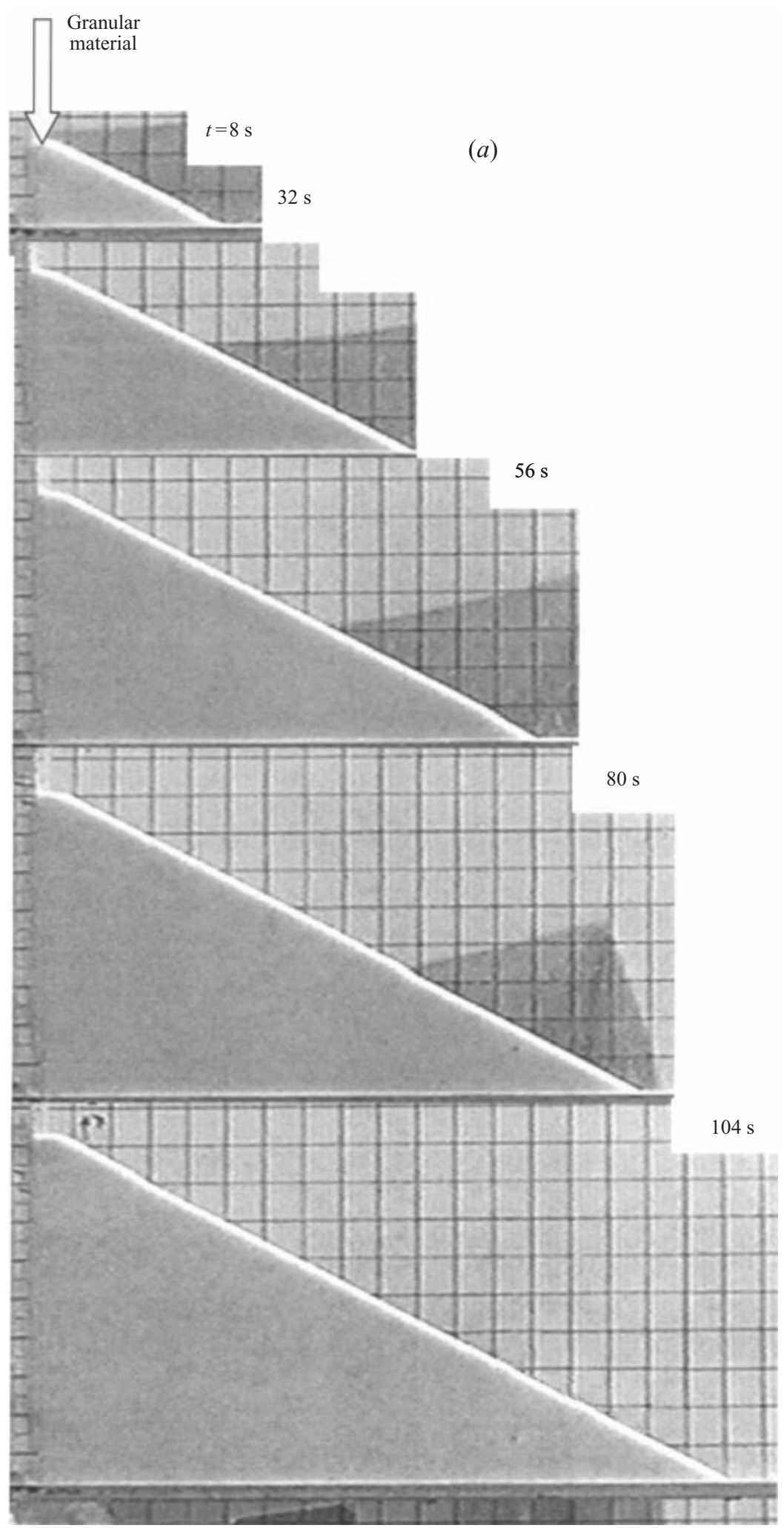

FIGURE 4. For caption see page 186. 


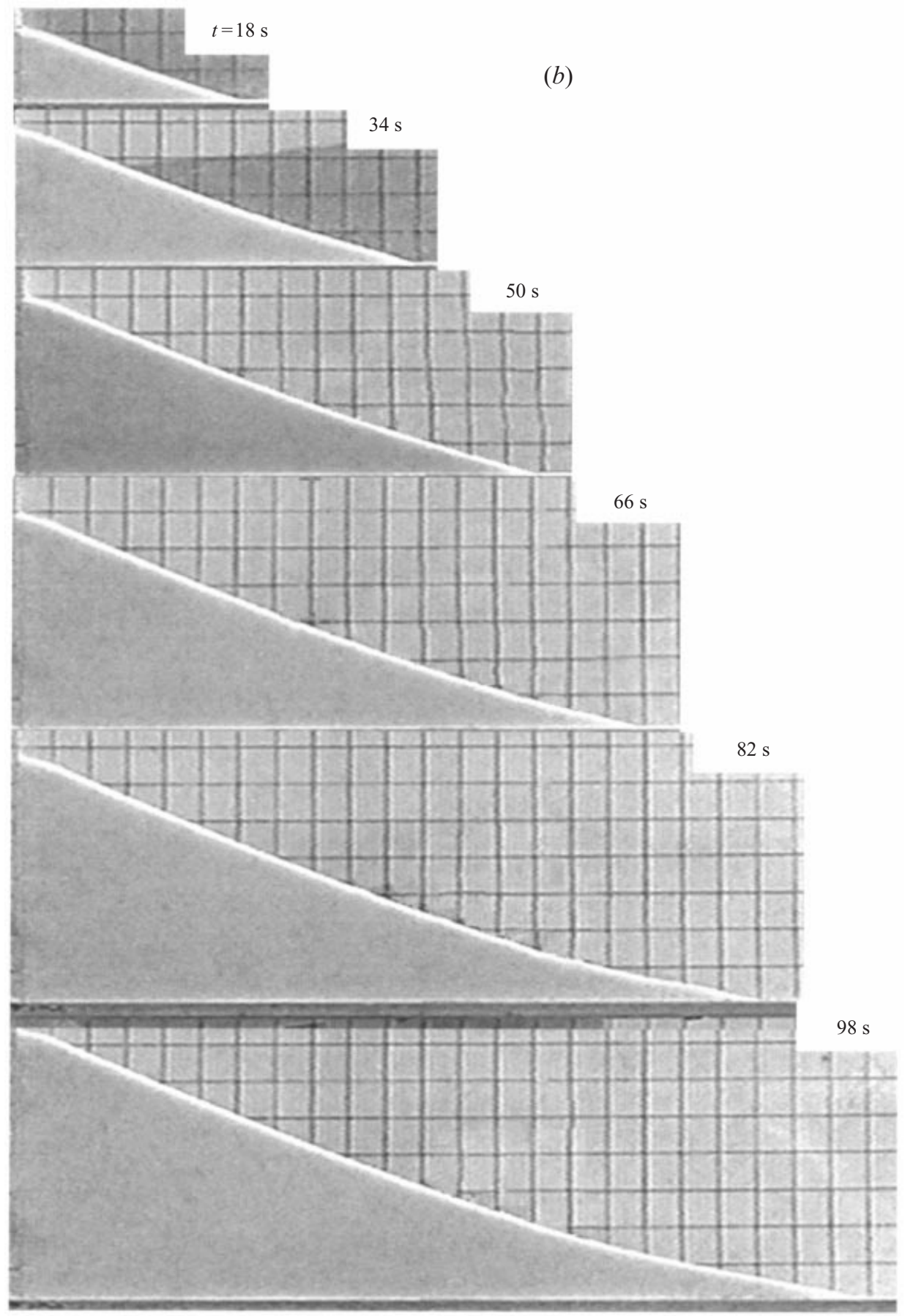

Figure 4. For caption see page 186. 

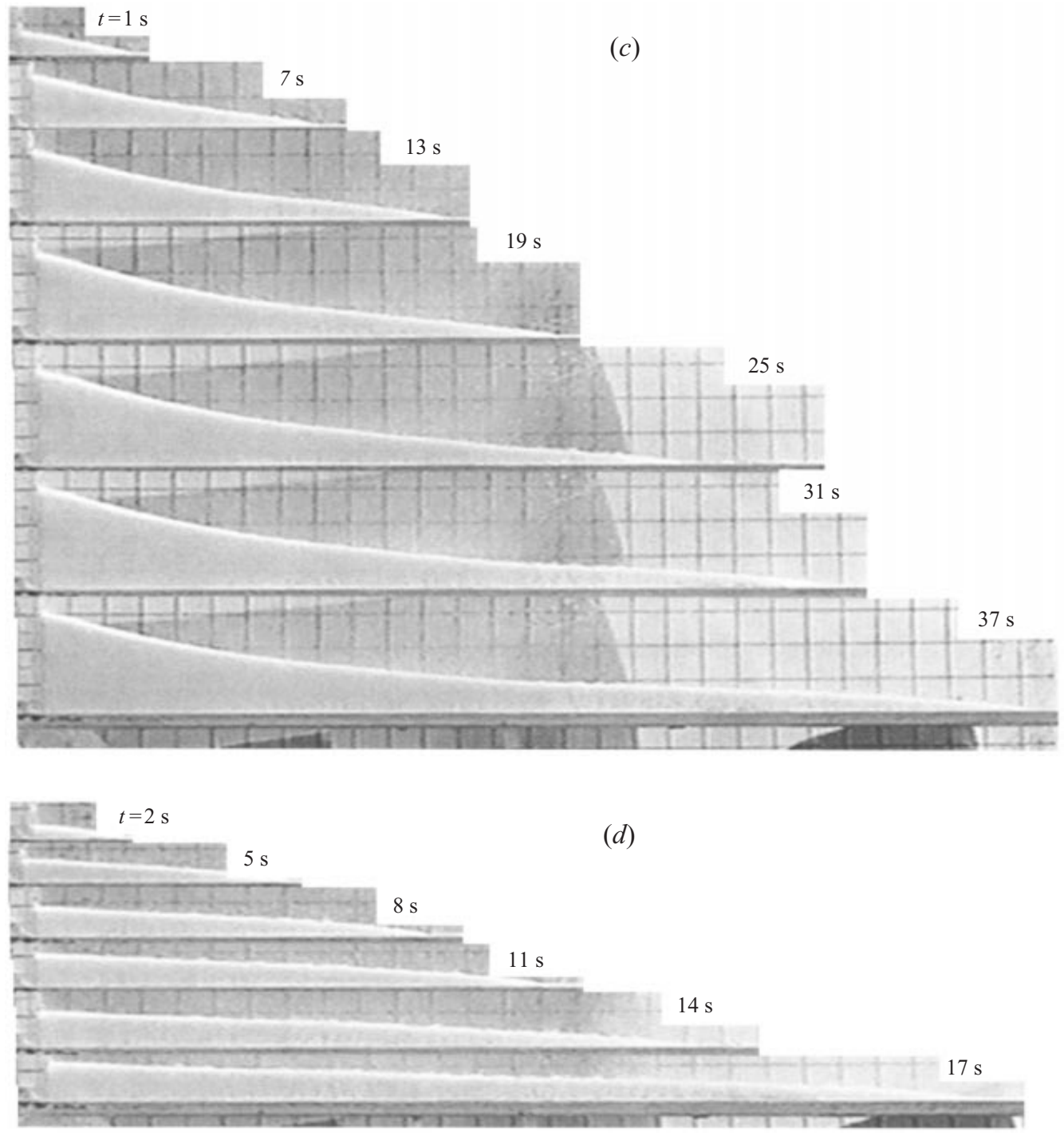

FIGURE 4. Sequences of images showing granular flow released at a rate $Q=0.88 \mathrm{~cm}^{2} \mathrm{~s}^{-1}$, at times $t$ (as shown) after the material is first released. (a) Planar flow in the absence of a fluidizing gas $\left(u_{g} / u_{m f}=0\right)$. (b) Aerated granular flow $\left(u_{g} / u_{m f}=0.42\right)$. (c) Aerated granular flow $\left(u_{g} / u_{m f}=0.84\right)$. (d) Fluidized granular flow $\left(u_{g} / u_{m f}=1.26\right)$.

illustrated by images from the planar bed in figure 4. Figure $4(a)$ shows the growth of a granular pile in the absence of a gas flow $\left(u_{g} / u_{m f}=0\right)$, where granular material is poured in at the left-hand side of the sequence of images. The pile evolves a wedge-shape with a uniform angle of repose except near the source. This is clearly shown in figure 5(a) where the shapes of the piles are normalized to a fixed length and height, and superimposed on one another. The pile is self-similar over the period of the experiment and the angle of repose constant.

When granular material poured onto a rigid surface is aerated, the shape of the developing pile of powder remains fairly self-similar, but the angle of repose is no longer constant and decreases from a maximum value of $\theta$ as the leading edge of 

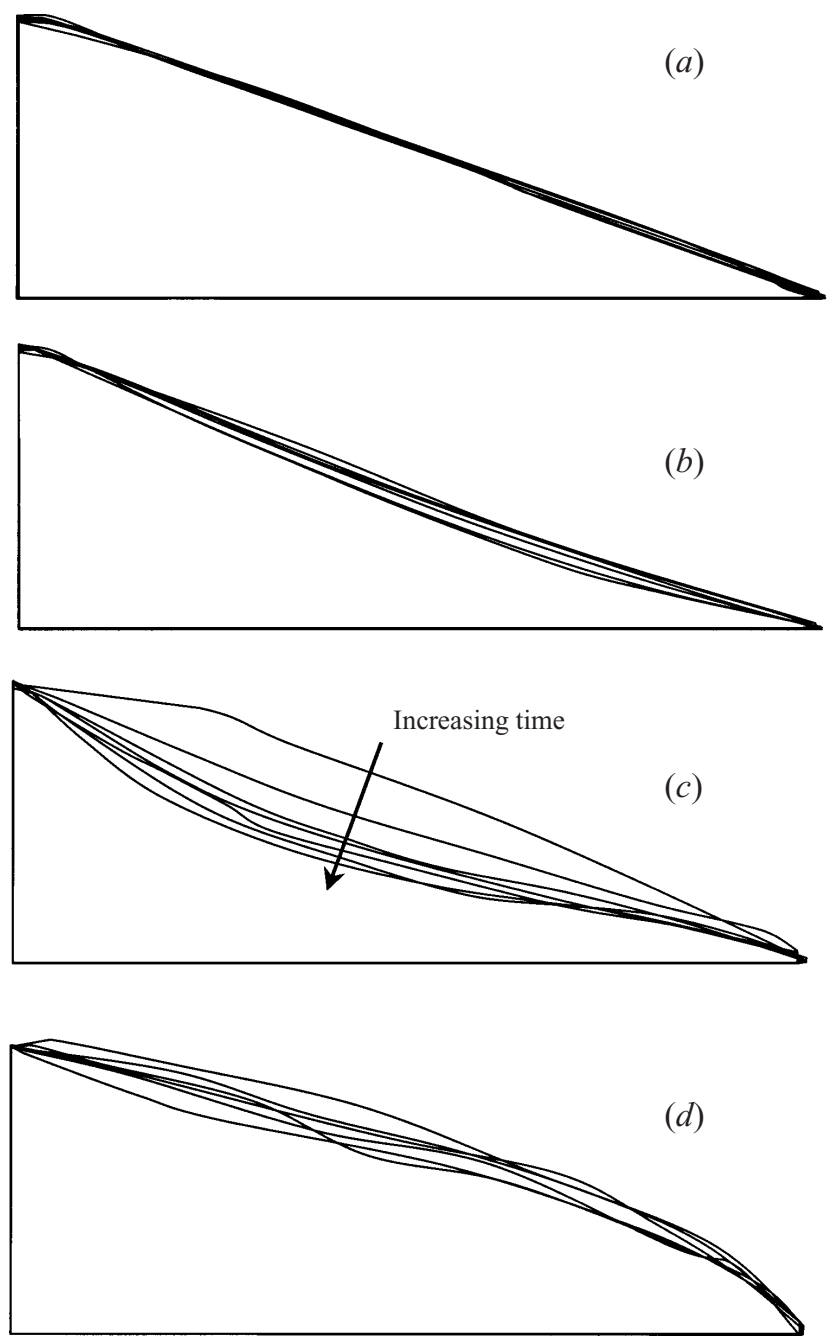

FIGURE 5. Profiles of the granular piles captured over a length of time and normalized with respect to pile length and height. The profiles $(a-d)$ correspond to the experiments shown in figure $4(a-d)$.

the moving pile is approached. This may be seen in figures $4(b)$ and $5(b)$ where $u_{g} / u_{m f}=0.42$. Through the use of marker particles within the bed, it was seen that the granular pile adjusted by material avalanching down the slope, and that the gas flow rate altered the position at which this initiated. When there is no gas flow the particles accumulate part way up the surface of the pile until their weight causes them to be pushed down the slope; but as the gas flow rate increases, the point at which this accumulation takes place moves down the slope until it reaches the leading edge of the pile. This distorts its conical shape, as shown in figures $4(b)$ and $5(b)$.

Figures $6(a)$ and $6(b)$ show shape predictions for $u_{g} / u_{m f}=0$ and $u_{g} / u_{m f}=0.42$ respectively using (27) to describe the angle of repose and (29) to describe the length of the pile. When $u_{g}=0$, agreement is good and any discrepancy is probably the result of uncertainty in the measurement of $Q$, the volume flux of material introduced 

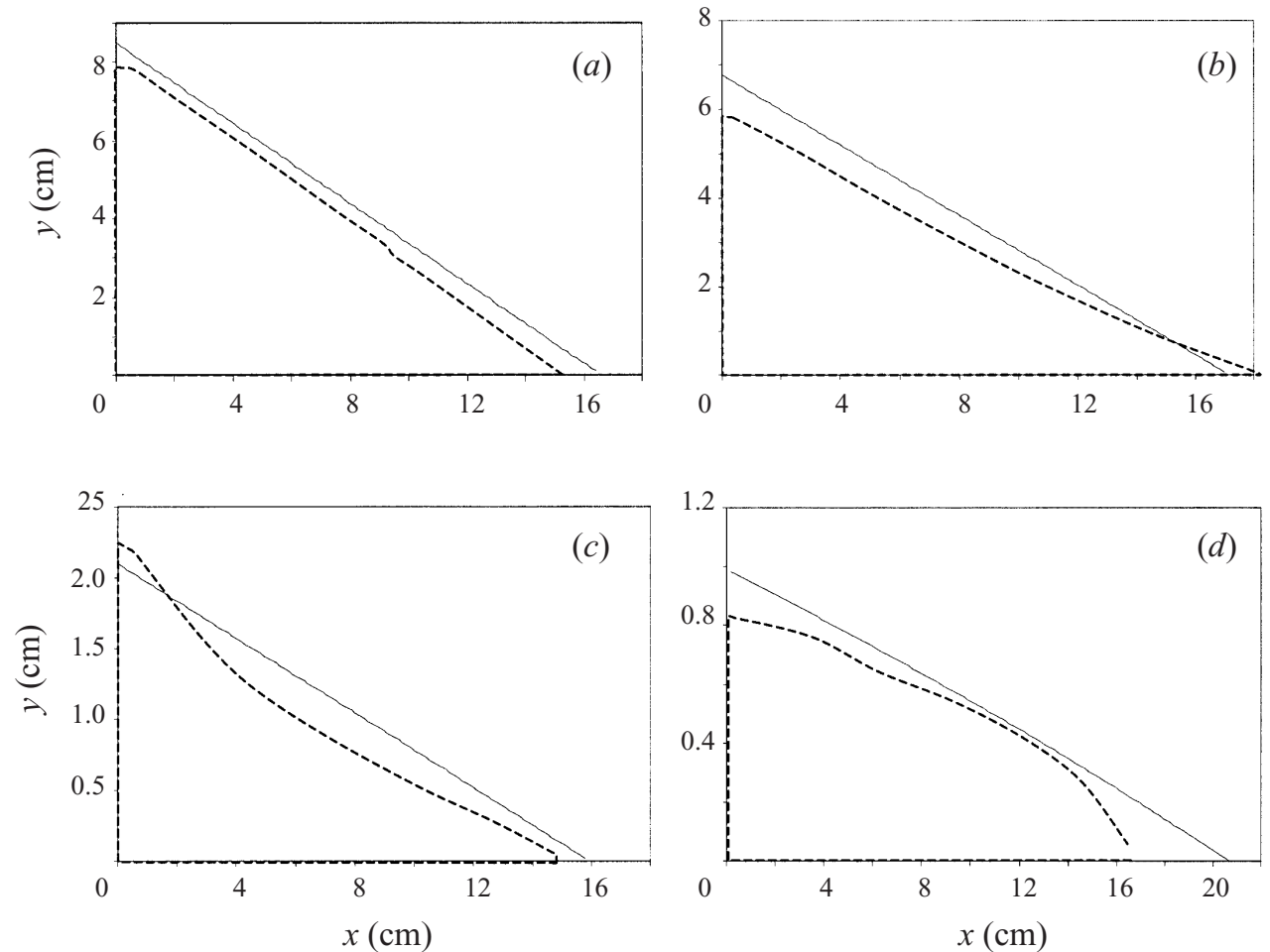

Figure 6. Comparison between measured profiles (dashed curves) and theoretical prediction (full curves): $(a),(b),(c)$ and $(d)$ correspond respectively to $u_{g} / u_{m f}=0,0.42,0.84$, and 1.26 , at times 80 , 66,19 and $11 \mathrm{~s}$ respectively, after material first introduced onto the wall. The theoretical prediction for $u_{g} / u_{m f}<1$ is based on (27) for predicting the angle of repose $\theta$ (with $C_{D}(R e) / C_{D}\left(R e_{m f}\right)=1$ ) and the length from (29). For $u_{g} / u_{m f}>1$, the shape is described by (35) and length by (34).

onto the wall. While the model will not capture the concavity at the leading edge of the pour when aerated, it does appear to successfully predict the shape of the rest of it.

When the gas flow rate approaches $u_{m f}$, the behaviour of the granular flow changes further. Figure $4(c)$ shows a granular pile when $u_{g} / u_{m f}=0.84$. The pile is much shallower and its top surface is more concave than at lower gas speeds, becoming more so with time as may be seen in figure $5(\mathrm{c})$. A careful examination shows that the nose of the pile is convex. Observation of marker particles within the pile indicates that there are two distinct regions: at the left-hand edge of the pour there is an immobile, aerated, conical pile of particles, and this is submerged in a flowing sheet of material. This sheet runs off the edge of the immobile region and forms a freeflowing stream to the right of the pile. Because of the inhomogeneous nature of this pour, we would not expect any one model to apply well. However, figure 6(c) shows that despite this, the extent and height of the pile of material is quite well predicted by (27) and (29). This suggests that despite its complex structure, the flow is dominated by interparticle friction and the effect of the interaction between the particles and gas upon it.

When $u_{g} / u_{m f}>1$, the shallow granular flow spreads rapidly along the rigid surface (figure $4 d$ ). Figure $5(d)$ shows that the pile is now convex in shape and appears to have a self-similar shape over the course of an experiment. The right-hand side of 

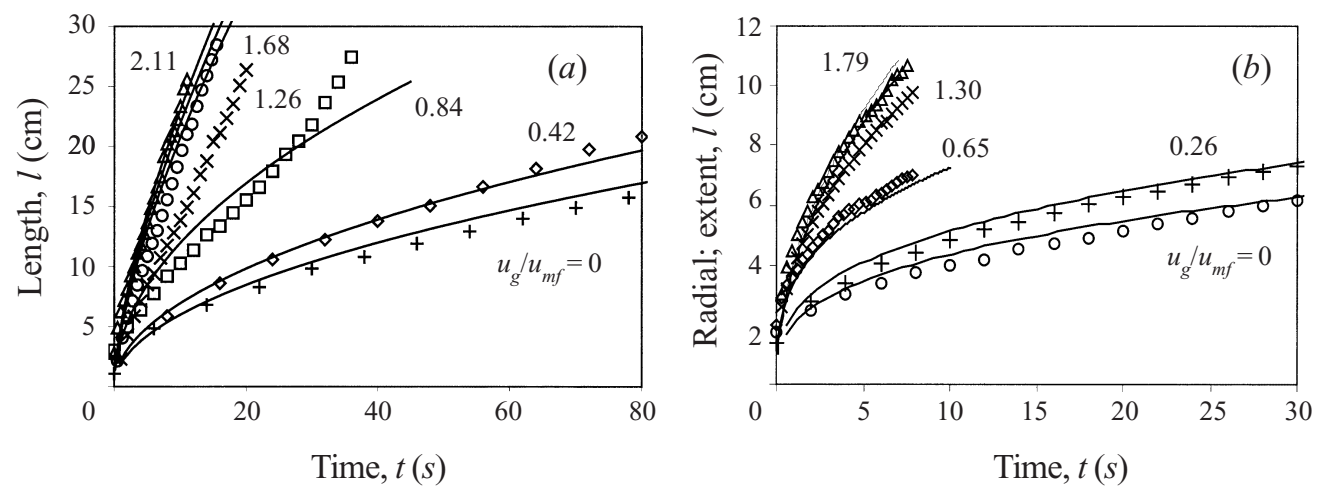

FIGURE 7. The encroachment distance of the granular layer as a function of time and gas flow rate for (a) planar flow with $Q=0.88 \mathrm{~cm}^{2} \mathrm{~s}^{-1}$, and $(b)$ axisymmetric flow with $Q=4.2 \mathrm{~cm}^{3} \mathrm{~s}^{-1}$. The different symbols correspond to different gas flow rates, as indicated on each figure. Theoretical predictions plotted for $u_{g} / u_{m f}<1$ are obtained from (29) using the angle of repose prediction (27) for planar flows, and (24) for axisymmetric flows. When $u_{g} / u_{m f}>1$, the theoretical predictions corresponding to (34) are plotted.

the granular flow in figure $4(c)$ is similar to that observed in figure $4(d)$, supporting the view that close to fluidization, e.g. $u_{g} / u_{m f}=0.84$, the pile consists of fluidized and aerated regions. Figure $4(d)$ corresponds to the case when the whole pile of material is fluidized and bulk movement occurs. Figure $6(d)$ shows the prediction of the shape described by equation (35). Again, the height and extent of the pile of material is predicted well, but the exact shape of the pour is not captured. Nevertheless, the spread of the particles appears to be dominated by the horizontal gradients of particle pressure and the effects of the drag force exerted by the gas flow.

\subsubsection{The rate of spreading of granular material}

The spread of the granular pile is critically dependent on the gas flow rate. The effect of aeration may be seen in figures $7(a)$ and $7(b)$ for a planar and axisymmetric flow respectively: as the gas flow rate is increased, the speed of propagation increases until $u_{m f}$ is reached when the rate of increase is reduced. When the granular material is aerated $\left(u_{g} / u_{m f}<1\right)$ the spread of a pile is described by (29) and when fluidized $\left(u_{g} / u_{m f}>1\right)$ by (34). Both expressions indicate that the spread of the material depends upon time $t$, gas flow rate $u_{g}$, and volume flux of particles $Q$. According to our continuum description, for fixed $Q$ and $u_{g}$ and $\alpha=1, l$ will vary as $t^{\frac{1}{n+1}}$ when aerated and as $t^{\frac{1}{n+2}}$ when fluidized. Figure 7 shows a comparison between theoretical and experimental measurements of the variation of the length of encroachment in the absence of a gas flow and when the bed is fluidized. The agreement between the predicted time exponents and those measured was confirmed by plotting the length of encroachment of the piles against time on a log-log plot. The accuracy of the predictions over a range of conditions is particularly good since there are no free parameters in the model.

The variation of $l$ with $Q$ for fixed values of $u_{g}$ and $t$ when the beds are fluidized is shown in figures $8(a)$ and $8(b)$ for planar and axisymmetric flows respectively. In both cases there is some scatter and at best satisfactory agreement. For the planar flows $l$ should vary as $Q^{1 / 3}$ and there is good agreement between theory and experiment for values of $Q$ up to $8 \mathrm{~cm}^{2} \mathrm{~s}^{-1}$, but there is significant underprediction $(\sim 30 \%)$ at higher values of $Q$. For an axisymmetric flow $l$ is predicted to vary according to $Q^{1 / 4}$. In this 


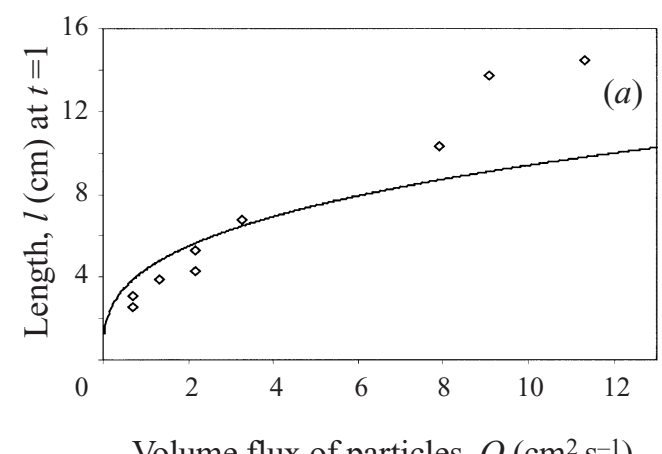

Volume flux of particles, $Q\left(\mathrm{~cm}^{2} \mathrm{~s}^{-1}\right)$

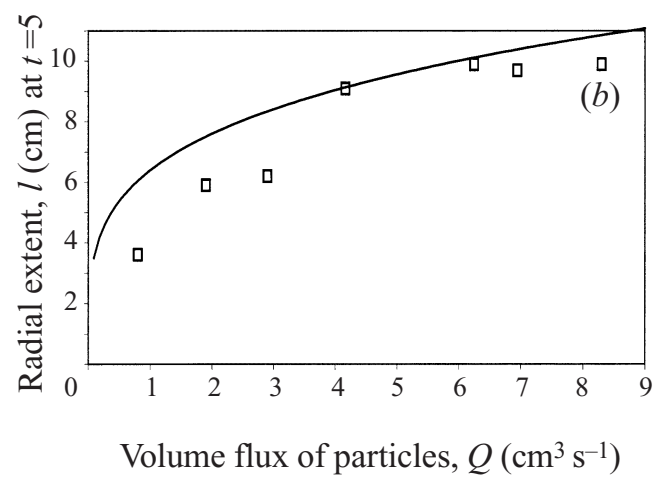

FIGURE 8. The effect of varying volume flux $Q$ on $(a)$ the length of a planar current $1 \mathrm{~s}$ after material is first released when gas flow rate is fixed at $u_{g} / u_{m f}=1.26$, and $(b)$ the radial extent of an axisymmetric granular flow $5 \mathrm{~s}$ after material is first released when gas flow rate is fixed at $u_{g} / u_{m f}=1.74$. The curves correspond to the theoretical prediction (34).

case agreement is reversed with a large difference between experiment and prediction at low values of $Q$ and good agreement at high values of $Q$.

The variation of $l$ with $u_{g}$ is shown in figures $9(a)$ and $9(b)$ for planar and axisymmetric flows respectively. The curves for several different values of $Q$ are shown, but for each curve the value of $l$ was measured at a time such that $Q t$ was a constant for all the experiments. Included on figures $9(a)$ and $9(b)$ are results from the numerical simulations and the quasi-static descriptions; for $u_{g}>u_{m f}$ the results of the numerical simulations are in agreement with the analytical expressions and for this reason are not included. According to the quasi-static description, the length $l$ is dependent on the volume of material poured in the pile $Q t$, and independent of the rate at which it is poured. The agreement between prediction and measurement is quite good when $u_{g} / u_{m f}<1$ and no systematic dependence on $Q$ was seen. As $u_{m f}$ is approached, the quasi-static description breaks down and predicts that the length tends asymptotically to infinity as friction tends to zero. The failure of the quasistatic description occurs because the characteristic time $t_{*}$, when frictional stresses are comparable to gradients of particle pressure and fluid drag, is larger than the time at which the length measurement was taken. Under these conditions, the dynamic description is strictly applicable and results obtained by solving numerically (21) are presented in figures $9(a)$ and $9(b)$. Experimental observations suggest that the flows appear to accommodate the transition between the aerated and fluidized regimes by becoming inhomogeneous and containing fluidized and non-fluidized regions. Above the minimum point of fluidization $l$ depends upon $Q$ when $Q t$ is fixed. This was observed, though agreement is less good than when $u_{g}<u_{m f}$; however, the theoretical and experimental curves are broadly similar if the disagreement between the predictions and measurements at some values of $Q$ is discounted (figure 8).

\section{Concluding remarks}

The effect of a vertical gas flow on the motion of a granular material over a horizontal surface has been investigated. A new vertically averaged description has been developed, extending previous analysis such as that of Savage \& Hutter (1989), by explicitly including a coupling between the gas and particle flows. Below the point of minimum fluidization, the effect of the gas flow is to reduce the angle of 

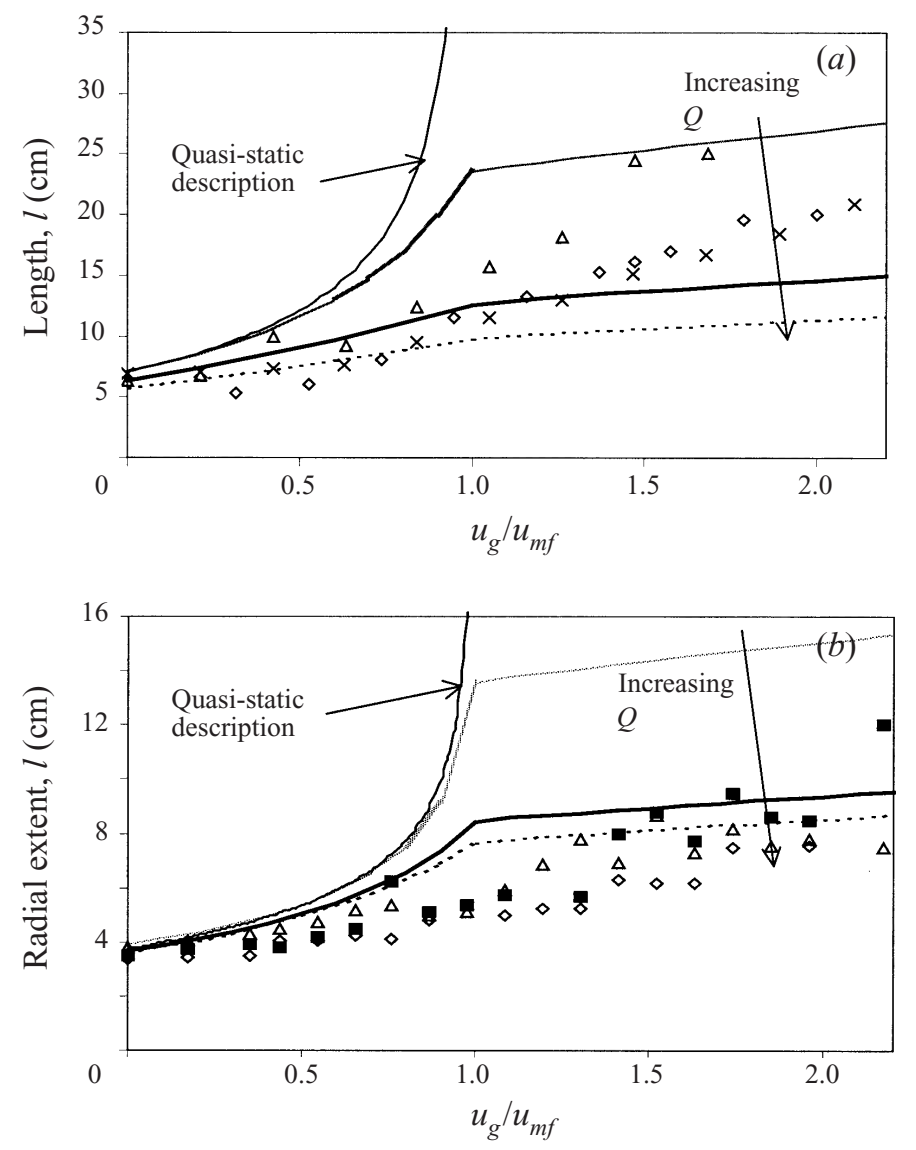

FIGURE 9. The effects of gas flow rate on encroachment distance for a fixed value of $Q t$ : $(a)$ planar flow with $Q t=11.9 \mathrm{~cm}^{2}$, with the symbols corresponding to experiments with $Q=0.88 \mathrm{~cm}^{2} \mathrm{~s}^{-1}$ $(\triangle), 5.7(\times), 11.9(\diamond) ;(b)$ axisymmetric flow with $Q t=25.4 \mathrm{~cm}^{3}$ and symbols corresponding to $Q=0.84 \mathrm{~cm}^{3} \mathrm{~s}^{-1}(\boldsymbol{\square}), 5.7(\diamond)$ and $8.3(\triangle)$. Also plotted on each figure is the quasi-static description given by (20) for $u_{g} / u_{m f}<1$. The results of numerical solutions to (21) are plotted for fixed values of $Q t$ corresponding to the experimental measurements. The analytical expressions for fluidized flows are not included in this figure because they are in accord with the numerical solutions of (21).

repose and a quasi-static description of this has been developed. Above the point of minimum fluidization a dynamic description has been developed from which a class of unsteady solutions has been obtained. An important feature of these models is that they do not contain any adjustable parameters, yet can describe reasonably well the variation of size and shape of the granular flow with time. The dynamic model of the fluidized granular flow is identical to the nonlinear advection-diffusion equation which describes buoyancy-driven flows in homogeneous porous media. This result may be expected because the drag force acts over the entire depth of the granular flow (where the particles are mobile) in the same manner as the drag on a buoyancydriven flow in a porous medium (where the particles are fixed). This suggests that the analogy of a flowing, dense fluidized bed with motion through a porous medium is stronger than that more usually adopted with a Newtonian fluid. 
A series of experiments was undertaken to test the theoretical descriptions. The models were in good qualitative agreement with the experimental measurements over a wide range of conditions. In addition, theoretical predictions were often quantitatively accurate without the use of any parameters which could not be measured directly. The agreement is sufficiently good to confirm that when inertia forces are weak $(\mathscr{R} \ll 1)$, as in our experiments, the horizontal momentum equation reduces to a balance between a horizontal gradient of particle pressure, friction stress on the horizontal wall, and the drag forces arising from particles moving in a gas flow. However, there were instances when the comparison was poor, notably with respect to particle flow rates when the particles were fluidized. The theoretical prediction is a simplified description of the granular flow dynamics and a number of physically relevant contributions to the stress are neglected in this treatment. The model assumes that the gas flow through the moving particles is the same as that expected through a static pile of particles, but a quickly moving granular flow will accelerate it, resulting in the particles moving faster than anticipated. This effect could be accounted for by solving the momentum equation describing the gas phase. In addition, to simplify our treatment, a viscous drag force was assumed and no account was taken of the sidewall friction for planar flows.

As well as instances where the terms within the model may be incomplete or inaccurate, there are two cases when the model itself is inappropriate. First, the model assumes that the flow is homogeneous and there are occasions when this is not true, particularly when $u_{g} \sim u_{m f}$. Secondly, when the particles are strongly fluidized the resulting layer of particles can be thin and the continuum assumption upon which the model is based may break down. There are a number of extensions to the analysis and experimental study which may be considered. The strong analogy between fluidized granular flows and buoyancy-driven flows in porous media (in the limit $\mathscr{R} \ll 1$ ) permits the model to be extended to include the effect of gravity-accelerated granular flows down slopes and the flow of internally vertically stratified granular flows along horizontal walls. The effect of shape and size distribution of granular material will affect both the fluidization process and the frictional drag, and this is currently being investigated.

I. E. gratefully acknowledges the Leverhulme Trust through the support of a Research Fellowship at Bristol University and EPSRC through the support of an Advanced Fellowship at UCL. Equipment support by the Royal Society is gratefully acknowledged. The UTHSCSA ImageTool program was developed at the University of Texas Health Science Centre at San Antonio and is available by anonymous FTP from maxrad6.uthscsa.edu.

\section{Appendix A}

A full treatment of the collisional stress term for the particulate phase requires an additional conservation equation for granular temperature $T$. Granular temperature, or particle kinetic energy, may be generated by particle-particle collisions, wall interactions, and the effect of a fluidizing gas flow. Below the conditions for fluidization $\left(u_{g} / u_{m f}<1\right)$ particles in a granular bed are typically locked together and their vibrational energy is low. However, when $u_{g} / u_{m f}>1$, particle mobility is greatly increased, though in the absence of bubbles there is very little particle vibration (Menon \& Durian 1997). Bubbles disturb the particles and significantly increase the granular temperature, and so the gas flow acts as a source for particle kinetic energy. 
This effect is represented by $Q_{g}$ which scales as $\rho_{g} u_{g}^{3} / H$. When this is added to the granular temperature conservation equation given by Nott \& Jackson (1992), which is then vertically averaged,

$$
\begin{aligned}
\underbrace{\frac{3}{2} \rho_{p}(1-\bar{\epsilon})\left(\frac{\partial \bar{T}}{\partial t}+\bar{u} \frac{\partial \bar{T}}{\partial x}\right)}_{O\left(\frac{\rho_{p} U T}{L}\right)}= & O \underbrace{\left.\frac{\rho_{p} T^{1 / 2} U^{2}}{H}, \frac{-\rho_{p} T^{3 / 2}}{H}\right)}-\underbrace{-\frac{\boldsymbol{q}_{p t} \cdot \hat{\boldsymbol{n}}}{H} \int_{0}^{H} \sigma_{s}: \nabla \boldsymbol{u} \mathrm{d} y}_{O\left(\frac{U T}{L}\right)} \\
& -\underbrace{\bar{I}}_{O\left(-\frac{\rho_{p} T^{3 / 2}}{d}\right)}) \quad \underbrace{\overline{Q_{g}}}_{O\left(\frac{\rho_{g} u_{g}^{3}}{H}\right)}
\end{aligned}
$$

where $\boldsymbol{q}_{p t}$ is the flux of pseudo-thermal energy into the granular flow and $I$ is the dissipation rate due to particle-particle collisions.

Granular flows are strongly dissipative and their granular temperature is maintained by a balance between generation and dissipation. The scaling analysis indicates that when $d \ll h$, as in the experiments, dissipation due to particle collisions at the wall will be negligible compared to that in the flow. From the point of view of generation of pseudo-thermal energy, two extremes may be considered depending on whether it is dominated by the wall or the gas flow. When production of pseudo-thermal energy at the wall is much larger than that in the flow, then by balancing the terms describing generation and dissipation in equation (A1), the pseudo-thermal energy is determined to be $T \sim U^{2} d / H$; this in turn requires $U / u_{g} \gg(H / d)^{1 / 6}\left(\rho_{g} / \rho_{p}\right)^{1 / 3}$. When pseudothermal energy production in the flow greatly exceeds that at the wall, then similarly $T \sim u_{g}^{2}(d / H)^{2 / 3}\left(\rho_{g} / \rho_{p}\right)^{1 / 3}$ and $U / u_{g} \ll(H / d)^{1 / 6}\left(\rho_{g} / \rho_{p}\right)^{1 / 3}$. In the experimental study described in this paper, the ratio $U / u_{g}$ is typically $O(H / L)$ and small for fluidized flows and $H / d$ is large, so that the gas flow is likely to be the principal source of granular stress. Thus, as the speed of the granular flow increases, the generation of pseudo-thermal energy by the wall dominates that of the fluidizing gas.

According to the analysis of Nott \& Jackson (1992), the stress at the wall is

$$
\hat{\boldsymbol{y}} \cdot \boldsymbol{\sigma} \cdot \hat{\boldsymbol{x}}+\mu N_{f}+\frac{\phi^{\prime} \sqrt{3} \pi \rho_{p}(1-\bar{\epsilon}) T^{1 / 2} U}{6\left(1-\epsilon_{0}\right)\left[1-\left((1-\bar{\epsilon}) /\left(1-\epsilon_{0}\right)\right)^{1 / 3}\right]}=0 .
$$

According to (A 2) and (18), the effect of collisional stresses generated by pseudothermal motion are negligible compared to frictional and drag forces when

$$
\rho_{p} T^{1 / 2} U \gg \frac{\rho_{p} g H\left|U-u_{g}\right|}{u_{m f}} .
$$

Substituting expressions for pseudo-thermal energy $T$ for the case when pseudothermal energy production is dominated by the gas flow results in

$$
\frac{U}{u_{g}} \ll \frac{g H}{u_{g}^{2}}\left(\frac{\rho_{p}}{\rho_{g}}\right)^{1 / 3}\left(\frac{H}{d}\right)^{1 / 3} \frac{\left|U-u_{g}\right|}{u_{m f}} .
$$

In the experiments described in this paper $U / u_{g} \sim O(1), H / d \sim 400, H \sim O(1) \mathrm{cm}$, $u_{g} \sim 10 \mathrm{~cm} \mathrm{~s}^{-1}$, and $\rho_{p} / \rho_{g}=2080$. Using these estimates for typical experimental values, the right-hand side of (A 4) is nearly 1000 times larger than the left-hand side, and so the inequality is clearly satisfied. 
When the production of pseudo-thermal energy is dominated by the wall rather than the gas flow (which, from above, is unlikely in the experiments), then substituting $T \sim U^{2} d / H$ gives

$$
\frac{U}{u_{g}} \gg\left(\frac{g H}{u_{g}^{2}}\right)^{1 / 2}\left(\frac{H}{d}\right)^{1 / 4} \frac{\left|U-u_{g}\right|}{u_{m f}} .
$$

In this case the right-hand side is about fifteen times smaller than the left-hand side, and so the inequality still holds.

Thus for the experiments considered in this paper, the collisional stress is negligible compared to drag and friction. This assumption is likely to become unrealistic when the granular flow is moving quickly compared with the speed of the gas flow through it, such as flow down a steep slope. In addition, the inequalities will break down if the particles in the flow are large compared with its depth; however, in such a case the use of a continuum model would also be questionable.

\section{Appendix B}

Let vertically averaged variables be denoted by

$$
\bar{f} h=\int_{0}^{h} f \mathrm{~d} y, \quad \bar{u}^{2} h=\beta \int_{0}^{h} u^{2} \mathrm{~d} y .
$$

The coefficient $\beta$ characterizes the velocity profile over the depth of the granular flow: for a parabolic velocity profile $\beta=\frac{6}{5}$, and for a plug flow $\beta=1$.

Integration of the equation describing the conservation of mass for the particulate phase, (6), yields

$$
\frac{\partial((1-\bar{\epsilon}) h)}{\partial t}=\left(1-\left.\epsilon\right|_{y=h}\right)\left[\frac{\partial h}{\partial t}+\left.u\right|_{y=h} \frac{\partial h}{\partial x}-\left.v\right|_{y=h}\right]-\frac{\partial}{\partial x}\left(\int_{0}^{h}(1-\epsilon) u \mathrm{~d} y\right),
$$

for a planar flow where $\boldsymbol{v}=(u, v)$. Application of the kinematic condition for the upper free surface of the granular flow yields

$$
\left.v\right|_{y=h}=\frac{\mathrm{D} h}{\mathrm{D} t}=\frac{\partial h}{\partial t}+\left.u\right|_{y=h} \frac{\partial h}{\partial x},
$$

and assuming that for a uniformly fluidized system the voidage is uniform, steady and is set by the local gas flow, so that $\partial \bar{\epsilon} / \partial t=\partial \bar{\epsilon} / \partial x=0$, equation (14) is obtained.

Integrating the inertial terms in the momentum equations across the fluid depth gives, for planar flows,

$$
\int_{0}^{h}\left(\frac{\partial h}{\partial t}+\boldsymbol{u} \cdot \nabla \boldsymbol{u}\right) \mathrm{d} y=\frac{\partial}{\partial t}(h \bar{u})+\frac{\partial}{\partial x}\left(\beta h \bar{u}^{2}\right)=\bar{u}\left[\frac{\partial h}{\partial t}+\frac{\partial}{\partial x}(h \bar{u})\right]+h\left[\frac{\partial \bar{u}}{\partial t}+\bar{u} \frac{\partial \bar{u}}{\partial x}\right],
$$

where the approximation $\beta=1$ has been invoked. The first term on the right-hand side of the above expression is identically zero from the conservation of mass (14). Vertically averaging the gradients of the stress tensor appearing in the horizontal momentum equation across the depth of the flow, likewise, yields

$$
\int_{0}^{h}\left(\frac{\partial \sigma_{x x}}{\partial x}+\frac{\partial \sigma_{x y}}{\partial y}\right) \mathrm{d} y=h \frac{\partial}{\partial x} \overline{\sigma_{x x}}+\frac{\partial h}{\partial x}\left(\overline{\sigma_{x x}}-\left.\sigma_{x x}\right|_{y=h}\right)+\left.\sigma_{x y}\right|_{y=0} ^{y=h}
$$


Under the long-wave approximation, horizontal gradients of quantities are significantly smaller than their vertical gradients, and the first term on the right-hand side of (B 2) is negligible compared to the last term. In addition, the second term on the right-hand side is negligible compared to the last term because the premultiplying factor $|\partial h / \partial x|$ is small compared to unity. Combining (B 1) and (B 2), in addition to vertically integrating the particle pressure and the drag exerted from the gas flow, yields (15).

\section{REFERENCES}

ANDERSON, K. \& JACKSON, R. 1992 A comparison of the solutions of some proposed equations of motion of granular materials for fully developed flow down inclined planes. J. Fluid Mech. 241, 145-168.

Anderson, K., Sundaresan, S. \& JaCKSON, R. 1995 Instabilities and the formation of bubbles in fluidized beds. J. Fluid Mech. 303, 327-366.

Bear, J. 1988 Dynamics of Fluids in Porous Media. Dover.

Brinkert, J. \& Davidson, J. 1993 Particle jets in fluidized beds. Trans. Inst. Chem. Engrs 71A, 334-336.

Brinkert, J. \& DAvidSon, J. 1995 Fluidized bed behaviour: a new collisional model. In Fluidization VIII (ed. J.-F. Large \& Laguérie), pp. 419-426. Engineering Foundation, Tours.

Davidson, J. F. \& Harrison, D. 1963 Fluidised Particles. Cambridge University Press.

DruitT, T. 1998 Pyroclastic density currents. In The Physics of Explosive Volcanic Eruptions (ed. J. Gilbert \& R. Sparks), vol. 145, pp. 145-182. The Geological Society.

GeldarT, D. 1986 Gas Fluidization Technology (ed. D. Geldart). John Wiley \& Sons Ltd.

Gilbertson, M. A. \& Yates, J. G. 1996 The tilting fluidized bed: a re-examination. Powder Tech. 89, 29-36.

Grace, J. 1970 The viscosity of fluidized beds. Can. J. Chem. Engng 48, 30-33.

Hopfinger, E. J. 1983 Snow avalanche motion and related phenomena. Ann. Rev. Fluid Mech. 15, $47-76$.

Huppert, H. E. \& Woods, A. W. 1995 Gravity-driven flows in porous layers. J. Fluid Mech. 292, 55-69.

JoHnsON, P. \& JACKSON, R. 1987 Frictional-collisional constitutive relations for granular materials, with application to plane shearing. J. Fluid Mech. 176, 67-93.

Johnson, P., NotT, P. \& JACKsON, R. 1990 Frictional-collisional equations of motion for particulate flows and their application to chutes. J. Fluid Mech. 210, 501-535.

Lun, C., Savage, S., Jeffrey, D. \& Chepurniy, N. 1984 Kinetic theories for granular flow: inelastic particles in couette flow and slightly inelastic particles in a general flow field. J. Fluid Mech. 140, 223-256.

Menon, N. \& Durian, D. J. 1997 Particle motions in a gas-fluidized bed of sand. Phys. Rev. Lett. 79, 3407-3410.

Molerus, O. 1982 Interpretation of Geldart's type A, B, C and D powders by taking into account interparticle cohesion forces. Powder Tech. 33, 81-87.

Nedderman, R. M. 1992 Statics and Kinematics of Granular Materials. Cambridge University Press.

NotT, P. \& JACKSON, R. 1992 Frictional-collisional equations of motion for granular materials and their application to flow in aerated chutes. J. Fluid Mech. 241, 125-144.

Savage, S. \& Hutter, K. 1989 The motion of a finite mass of granular material down a rough incline. J. Fluid Mech. 199, 177-215.

Tsuji, Y., TANAKA, T. \& IsHida, T. 1992 Lagrangian numerical simulation of plug flow of cohesionless particles in a horizontal pipe. Powder Tech. 71, 239-250. 